\title{
Weakly stratified laminar flow past normal flat plates
}

\author{
By IAN P. CASTRO \\ School of Engineering Sciences, University of Southampton, \\ Highfield, Southampton SO17 1BJ, UK
}

(Received 23 May 2001 and in revised form 29 August 2001)

Numerical computations of the steady, two-dimensional, incompressible, uniform velocity but stably stratified flow past a normal flat plate (of unit half-width) in a channel are presented. Attention is restricted to cases in which the stratification is weak enough to avoid occurrence of the gravity wave motions familiar in more strongly stratified flows over obstacles. The nature of the flow is explored for channel half-widths, $H$, in the range $5 \leqslant H \leqslant 100$, for Reynolds numbers, $R e$, (based on body half-width and the upstream velocity, $U$ ) up to 600 and for stratification levels between zero (i.e. neutral flow) and the limit set by the first appearance of waves. The fourth parameter governing the flow is the Schmidt number, $S c$, the ratio of the molecular diffusion of the agent providing the stratification to the molecular viscosity. For cases of very large (in the limit, infinite) $S c$ a novel technique is used, which avoids solving the density equation explicitly. Results are compared with the implications of the asymptotic theory of Chernyshenko \& Castro (1996) and with earlier computations of neutral flows over both flat plates and circular cylinders. The qualitative behaviour in the various flow regimes identified by the theory is demonstrated, but it is also shown that in some cases a flow zone additional to those identified by the theory appears and that, in any case, precise agreement would, for most regimes, require very much higher $R e$ and/or $H$. Some examples of multiple (i.e. non-unique) solutions are shown and we discuss the likelihood of these being genuine, rather than an artefact of the numerical scheme.

\section{Introduction}

Steady flow around a two-dimensional bluff body is a classical problem in fluid mechanics. As the body Reynolds number increases from zero it is well-known that the near wake becomes unsteady at a relatively low Reynolds number (based on upstream velocity and body scale but dependent on body shape) and laminar vortex shedding begins well before any transition to turbulence in the separated shear layers. A somewhat esoteric but interesting class of laminar flow is that in which the (Kármán-type, asymmetric, periodic) vortex shedding is prevented by the imposition of symmetry on the wake centreline, either by the physical addition of a central splitter plate or, analytically or numerically, by simple enforcement of a frictionless boundary condition. The behaviour of such flows has received considerable attention, starting with the free-streamline models of Helmholtz (1868) and Kirchhoff (1869), in which the flow within the separated region immediately behind the body was supposed stagnant and the downstream nature of the flow was not explored. An alternative model was proposed by Batchelor (1956), in which the separated wake 
was supposed to consist of two regions of equal and opposite (constant) vorticity either side of the symmetry axis. Subsequent numerical computations (for cylinders, Fornberg 1985) and analytical studies (e.g. Peregrine 1985; Smith 1985) suggested that there was some truth in both these models, but the full asymptotic nature of the flow was only finally revealed by Chernyshenko (1988). It turned out that in the region very close to the body the flow is essentially Kirchhoff-like, but with almost constant vorticity regions further downstream in the two counter-rotating eddies on either side of the symmetry axis. Contrary to both the earlier models, however, the wake was found to be unbounded in both width, $W$, and length, $L$, with $W, L=O(R e)$ so that the wake vorticity is $O\left(R e^{-1}\right)$ in the limit $R e \rightarrow \infty$. It should be noted that the first theory in which both $L$ and $W$ were of order Re was that of Taganov $(1968,1970)$, although some of his quantitative results differ from those of Chernyshenko (1988), as discussed in the latter and by Chernyshenko \& Castro (1996 hereinafter denoted as CC). Throughout the present work the Reynolds number is defined using the (unit) body half-width and the upstream velocity. This is consistent with a number of more recent papers, but means that, for an equivalent flow, $R e$ values are effectively larger by a factor of two than those of Fornberg $(1985,1991)$, who used the cylinder diameter as the length scale; i.e. $R e_{\text {here }}=\frac{1}{2} R e_{\text {Fornberg }}$.

The problem becomes a little more realistic if the body is effectively just one of an infinite cascade of identical bodies, by being located at the centre of a plane channel (as in the case of a body in a wind tunnel, for example). This imposes a further parameter, defined here as $H$, the ratio of the channel width to the body width. Numerical solutions for circular cylinder cascades have been presented by Fornberg (1991) (with $R e \leqslant 400$ in the present terms and $5 \leqslant H \leqslant 100$ ) and for flat plate cascades by Natarajan, Fornberg \& Acrivos (1993) (with $R e \leqslant 400$ and $5 \leqslant H \leqslant 25$ ) and Ingham, Tang \& Morton (1990) (with $R e \leqslant 500$ and $H=2$ ). Chernyshenko's (1988) asymptotic theory was extended to this case by Chernyshenko \& Castro (1993) and, again, showed how the numerical results tend qualitatively towards those expected, but only at the very highest Reynolds numbers.

The latter asymptotic theory was extended further by CC to the case in which the additional physical effect of stable density stratification was added. This introduces two additional parameters: a Richardson number, $R i$, defined by $-\left[\left(g H^{2} / \rho U^{2}\right) \partial \rho / \partial y\right]$ and the Schmidt number, $S c$ (the ratio of the coefficient of diffusivity of the agent providing the density variations to the kinematic viscosity). The density gradient, $\partial \rho / \partial y$, refers to conditions far upstream and is assumed small enough to allow the Boussinesq approximation to be applied. Weak stratification is here defined by the $R i$ range $0 \leqslant R i \leqslant \pi^{2}$; for larger values the flow becomes subcritical for at least one wave mode and disturbances can then propagate upstream without limit (if the effects of viscosity are ignored). A more convenient measure of the strength of the stratification is provided by the parameter $K$, defined by $K=R i / \pi^{2}$, so that the range of interest in this work is limited to $0 \leqslant K \leqslant 1$ with $K=0$ and $K=1$ referring to neutral and 'critical' conditions, respectively. With velocities normalized by $U$ and pressure normalized by $\rho U^{2}$ the flow is governed by the following equations:

$$
\begin{gathered}
\boldsymbol{u} \cdot \nabla \boldsymbol{u}=-\nabla p+(1 / R e) \nabla^{2} \boldsymbol{u}-(R i \rho / H) \boldsymbol{j} \\
\boldsymbol{u} \cdot \nabla \rho=(1 /(\operatorname{ReS} c)) \nabla^{2} \rho, \\
\nabla \cdot \boldsymbol{u}=0 .
\end{gathered}
$$

Here $\rho$ is the density variation normalized by the upstream quantity $\partial \rho / \partial y$ and for brevity is called the density in what follows. Symmetry about $y=0$, the $x$-axis through 


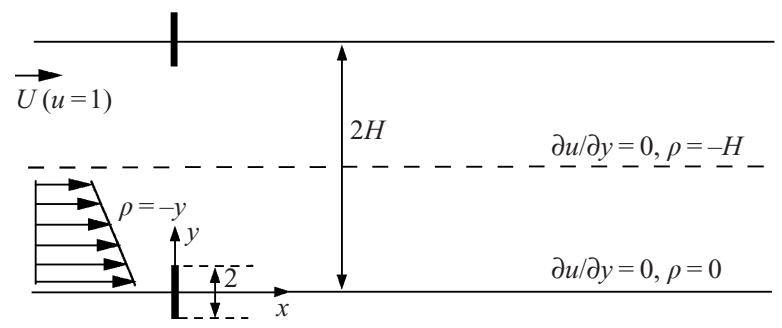

FIGURE 1 . Sketch of the geometry. Only the half-space $0<y<H$ is computed.

the centre of the body, allows us to consider only the upper half of the flow field. The boundary conditions used in CC and in the present numerical computations (see later) are

$$
\begin{gathered}
x \rightarrow-\infty, \quad u \rightarrow 1, \quad \rho \rightarrow-y, \quad \partial u / \partial y=0, \\
y=0, \quad \partial \boldsymbol{u} / \partial y=0, \quad \rho=0 \quad \text { outside the body, } \\
y=H, \quad \partial \boldsymbol{u} / \partial y=0, \quad \rho=-H,
\end{gathered}
$$

and, on the body surface,

$$
\boldsymbol{u}=0, \quad \partial \rho / \partial n=0 .
$$

Equation (1.5) is the imposed symmetry condition and (1.6) is the zero-stress condition ensuring that $y=H$ is a streamline with a condition on $\rho$ consistent with (1.4). Figure 1 is a sketch of the problem considered. Since, as discussed later and in $\mathrm{CC}$, stratification effectively reduces the Reynolds number at which the asymptotic results are likely to be accurate, the major motivation of the present work was to undertake a much more comprehensive numerical study of this case than has been done previously.

Before proceeding, in $\S 2$, to outline the numerical techniques used and then, in $\S 3$, to present the results, it is helpful to summarize the qualitative behaviour of the flow in the various regimes first identified for the neutral case (i.e. no density stratification) by Chernyshenko (1988) and in CC for the stratified case. First, for $R e \rightarrow \infty$ but with $H=O(R e)$ both the eddy length and width have order $R e$. The situation is sketched in figure 2(a). The flow on the body scale is the Kirchhoff free-streamline flow, with the downstream eddy effectively open and extending to infinity. On the eddy scale, however, the body shrinks to a point, the limiting flow is inviscid and is, in fact, the Sadovskii flow in a channel, delineated by Chernyshenko (1993) and Turfus (1993). This flow contains two closed-streamline regions touching on the symmetry axis and with constant (but oppositely signed) vorticity within them-as required by the Prandtl-Batchelor theorem (Batchelor 1956). The Bernoulli jump in velocity across the bounding streamline is zero, as shown by CC. Unbounded (i.e. $H=\infty$ ) Sadovskii flows of this sort have been computed numerically by Sadovskii (1970) and Saffman \& Tanveer (1982). The major flow characteristics - body drag, eddy vorticity, eddy length and eddy width - can be expressed by the following relations:

$$
\begin{gathered}
C_{d} R e=\alpha\left(H /\left(k_{d}^{2} R e\right), R i\right), \\
\omega_{e d} k_{d}^{2} R e=\beta\left(H /\left(k_{d}^{2} R e\right), R i\right), \\
L /\left(k_{d}^{2} R e\right)=\gamma\left(H /\left(k_{d}^{2} R e\right), R i\right), \\
W / L=\delta\left(H /\left(k_{d}^{2} R e\right), R i\right) .
\end{gathered}
$$




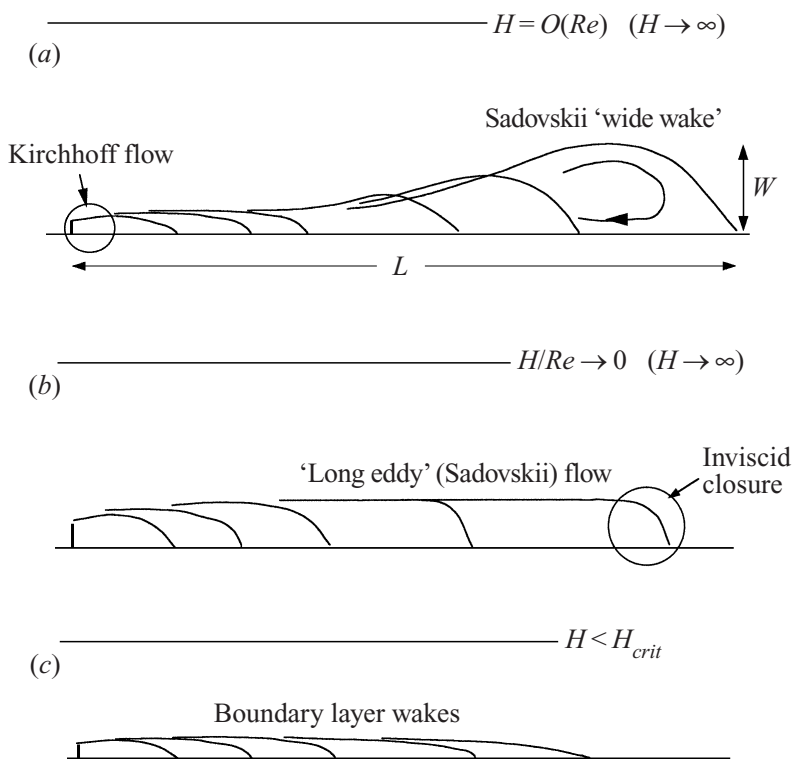

FIGURE 2. Flow regimes for asymptotically large Reynolds number.

$k_{d}$ is the usual Kirchhoff drag coefficient ( 0.88 for a flat plate in unbounded flow) and the four functions, $\alpha, \beta, \gamma$ and $\delta$, depend only on the particular Sadovskii flow resulting from the given values of $H /\left(k_{d}^{2} R e\right)$ and $R i$. (Details of how this can be calculated are given in CC.) Note also that these results were worked out for the limiting case in which $S c \rightarrow \infty$, but were argued in CC not to depend strongly on the particular value of $S c$. We address this particular issue when discussing the present numerical results.

Secondly, we consider the situation in which both $R e \rightarrow \infty$ and $H \rightarrow \infty$ but in such a way that $H / R e \rightarrow 0$. In this case a 'long eddy Sadovskii flow' emerges, illustrated in figure 2(b) and first identified by Chernyshenko (1993). Apart from the Kirchhoff region at the body and the inviscid eddy closure around $L$, the eddy has essentially constant width and vorticity and the parameters have the following functional forms:

$$
\begin{gathered}
C_{d}=\alpha_{2}(R i) k_{d}^{2} / H, \\
\omega_{e d}=\beta_{2}(R i) / H, \\
L=\gamma_{2}(R i) k_{d}^{2} R e, \\
W=\delta_{2}(R i) H .
\end{gathered}
$$

Note the conclusion that neither the drag nor the eddy vorticity depend on $R e$ and the eddy length does not depend on $H$. Again, the four functions (constant at fixed $R i$ ) can also be determined from the theory presented in CC. It should be emphasized that this long-eddy solution, for which CC presented some apparent numerical confirmations (but see later), is strictly only valid as $H \rightarrow \infty$ and will thus only emerge for a large enough channel width. If $H$ is too small, the four 'constants' will probably be functions also of $H$ and the asymptotic theory makes no predictions about their behaviour or, indeed, about the detailed nature of the flow in such cases. Actually, for small enough $H$ the boundary conditions probably do not admit anything other than long, thin 'boundary layer-type wakes', as indicated in figure 2(c), with viscous closure around $x=L$. This possibility was discussed briefly by Chernyshenko \& Castro (1993) and, 
as they noted, is supported by the numerical calculations of Milos \& Acrivos (1986) and Milos, Acrivos \& Kim (1987). These issues are discussed further in due course.

The influence of the stratification on the separated wake depends critically on the channel width. For large enough $H$ (strictly, large enough $H /\left(k_{d}^{2} R e\right)$ ) the stratification scale is large compared with the eddy scale so that the effects are not large. Stratification reduces the drag and the eddy vorticity $\left(\alpha_{2}\right.$ and $\beta_{2}$ are decreasing functions of $R i$ ), while increasing the eddy length ( $\gamma_{2}$ increasing with $R i$ ). For narrow channels, on the other hand, the changes in drag and eddy vorticity are much larger and $L$ reduces significantly. So at intermediate $H$, changes in $L$ may be non-monotonic at fixed $R e$ and $H$. In neutral flow the vorticity shed into the eddy at the body is lost by diffusion from the eddy, through the vorticity boundary layers surrounding it. For $R i>0$, however, in addition to the vorticity generation by the body, vorticity is generated baroclinically outside the eddy and can thus diffuse into it. The overall vorticity balance is quite subtle and the diffusion of the additional externally produced vorticity is insufficient to prevent a much larger fall in $\omega_{e d}$ for smaller channels. Some preliminary numerical computations were presented in $\mathrm{CC}$ and were shown to have some features at least qualitatively consistent with the above aspects of the theory. They therefore suggested that the physical mechanisms identified therein were essentially correct. However, they were very limited in scope; values of $H$ and $R e$ were not high enough for the appearance of clear examples of the 'wide wake' flows sketched in figure 2(a), for example, and the grids used were too coarse to allow adequate resolution of the density boundary layers for the value of $S c$ used (1000). It is the aim of the present paper to present a more comprehensive set of computations, covering a wider parameter range than was possible earlier. In some respects the results corroborate the earlier work. However, they also suggest the existence of intermediate flow regimes currently not identified by any theoretical treatment and thus lead to further interesting questions.

\section{The numerical techniques}

Equations (1.1)-(1.3) were discretized using a finite volume approach on a staggered, rectangular, non-uniform grid and solved using a multigrid version of a fairly standard iterative (ADI) method. The multigrid version of the code was developed from an earlier NS solver of the author's and is described in detail by Paisley (1997). It is worth noting that the code also contains the option of conducting genuine (second-order accurate) time-dependent calculations, but only the steady flow (iterative) option was used for the present work. (Further comments on this point are given later). Brief details of the entire (steady-flow) scheme are provided here for completeness.

Integration of the momentum and density equations (1.1) and (1.2) over a typical control volume, with use of the divergence theorem, gives

$$
\sum\left[\left(u \phi-\Gamma \frac{\partial \phi}{\partial x}\right) \mathrm{d} y+\left(v \phi-\Gamma \frac{\partial \phi}{\partial y}\right) \mathrm{d} x\right]=S^{\phi} \mathrm{d} x \mathrm{~d} y .
$$

$\phi$ stands for $u, v$ or $\rho, \Gamma=1 / \operatorname{Re}$ or, for the density equation, $1 /(\operatorname{Re} S c)$ and $S^{\phi}$ is a source term. In the momentum equations this contains the pressure gradient plus, for the vertical momentum equation, the buoyancy term. For the density equation, $S^{\phi}=0$. Central differencing was used for the diffusive terms. Solution accuracy is crucially dependent on the way in which the advective terms are approximated. In this work a monotonic scheme was used, which prevents unphysical oscillations and was derived by assuming that the flow across cell boundaries is one-dimensional in directions 
normal to the flow. It is based on a scheme similar to the one originally devised for transient, compressible flows (van Leer 1974) and is described by Leonard \& Mokhtari (1990). A mixture of central differencing and second-order upwind interpolation is used. Suppose that the value of the flow variable at the centre of a cell is $\phi_{P}$ and that $\phi_{U}$ and $\phi_{D}$ denote the corresponding values at the cells immediately upstream and downstream, respectively. The value of $\phi$ at the downwind cell face, $\phi_{f}$, midway between the $P$ and $D$ points (required, for example, for the $u \phi$ product in the first term of (2.1)) is given by

$$
\phi_{f}=\frac{1}{2}\left(\phi_{P}+\phi_{D}\right) \hat{\phi}_{P}+\frac{1}{2}\left(3 \phi_{P}-\phi_{U}\right)\left(1-\hat{\phi}_{P}\right),
$$

where $\hat{\phi}_{P}$ is the ratio

$$
\hat{\phi}_{P}=\frac{\phi_{P}-\phi_{U}}{\phi_{D}-\phi_{U}} .
$$

The convective and diffusive contributions from the four cell faces are collected in the usual way so that (2.1) can be expressed as

$$
a_{P} \phi_{P}=\sum a_{m} \phi_{m}+S_{P}^{\phi},
$$

where the summation is taken over the values at the centres of the four neighbouring cells and the multiplying coefficients $\left(a_{P}\right.$, etc.) contain the convective and diffusive flow rates. The source term contains the second-order corrections from the convective scheme just described. These equations were solved iteratively in turn (i.e. for each variable sequentially), along with a pressure perturbation equation (derived using (1.3)), using SIMPLER (Patankar 1980). This is a more efficient version of the wellknown SIMPLE algorithm. Under-relaxation on the velocities, the density and the pressure perturbation was required, as usual for such schemes.

For flows in which $S c \gg 1$, solution of the density equation (1.2) would require a much finer grid than that necessary for the momentum equations, in order to capture the density boundary-layer regions adequately. This was not done in $\mathrm{CC}$, where numerical solutions were presented for cases with $S c=1000$ (roughly equivalent to situations in which the stratification is provided by, say, salt in water). As indicated earlier, some questions therefore arise over the accuracy of the solutions. In the present work an entirely different technique was used, which is actually exact for $S c=\infty$. In that specific case, the density field reduces to

$$
\left.\begin{array}{ll}
\rho=\rho(\psi)=-\psi, & \psi>0, \\
\rho=\rho(\psi)=0, & \psi \leqslant 0,
\end{array}\right\}
$$

instead of (1.2) and where $\psi$ is the stream function. Cases with $S c=\infty$ were obtained by avoiding solution of (1.2) altogether and, rather, computing $\psi$ after each velocity field iteration and then setting the density field according to (2.4) before proceeding with the next iteration. $\psi$ was determined via a simple quadrature scheme; this was significantly faster than the time required to solve the full density equation and also, of course, was much more accurate than the latter. $S c=O(1)$ cases were solved in the usual way by including solution of the discretized version of (1.2) in the iteration process. The results presented in $\S 3$ include assessment of the differences in the solutions for the $S c=1$ and $S c=\infty$ cases. (Recall that, as stated earlier, CC argued that the asymptotic results-worked out for $S c=\infty$-should not, in fact, depend too strongly on the particular value of $S c$.)

For multigrid solution (an almost exclusively used option for the present computations) a hierarchy of grids (up to five levels) was defined, with successively coarser 
grids being produced by simply removing every other node in both directions. Continuity control volumes on the coarse grid were formed from the sum of the four control volumes on the next finer grid. Variables were restricted to the coarser grid using averages of the nearest fine grid neighbours, area weighted to ensure preservation of mass fluxes on non-uniform grids. Bilinear interpolation was used for prolongation of coarse grid solutions back onto the next finer grid. Generally, a W-cycle was used to visit the grids in the hierarchy, with one pre-smoothing and one or two post-smoothing iterations on each. As usual in the multigrid approach, in principle only the coarsest grid equations needed to be solved with any accuracy - the role of the solver on the other grids is essentially to smooth errors.

For the present problem, particular care was needed in the generation of the grids, to ensure that appropriate cell structure was maintained around the flat plate on each member of the grid hierarchy. Furthermore, because of the staggered grid arrangement, various adjustments to the coefficients in (2.3) were necessary for cells adjacent to the plate. For example, $u$-velocity cells spanned the plate, allowing straightforward imposition of the plate boundary condition $(u=0)$, but requiring a de-coupling of the conditions within those cells on either side of the plate and other modifications at the plate tip, which was located in the centre of a $u$-cell. Similarly, the $v$-velocity cells lay adjacent to the plate (on each side), so zero-flux conditions had to be applied on the appropriate faces. Pressure nodes were not located on the plate surface so there were none of the difficulties which would otherwise arise if a node coincided with the plate tip. Other boundary conditions simulated those used in the asymptotic theory of CC, except that zero-gradient conditions were imposed at the outlet. The question of precisely where this outlet, and the inlet, need to be located in order to obtain results essentially independent of their location is discussed below; it turns out to be strongly dependent on the degree of stratification.

A typical (fine) grid had $642 \times 162$ nodes in the $x$ - and $y$-directions, respectively, so with four levels of coarsening the coarsest grid had some $42 \times 12$ nodes; the factors are not precisely 16 because of the need to maintain grid numbers equal to integral multiples of two on each side of the plate. Even larger grids were used in many cases (see table 1 for an example). The speed-up factors for convergence (deemed to have occurred when the sum of (absolute) residuals on each equation fell below $10^{-4}$ ) was very significant and generally consistent with those found by Paisley (1997), who showed that, on a $320 \times 40$ node grid, convergence in single-grid mode took some 30 times longer than when using a five-level multigrid option. For some of the present cases even greater efficiencies were achieved because convergence rates were essentially independent of the grid size and much finer grids were used than those of Paisley. Not surprisingly, however, convergence rates became slower with increasing $R e$ or $R i$. Increasing $R i$, in particular, led to greater convergence difficulties at the higher $R e$ values because of the greater coupling between the velocity and density fields. The difficulties were most severe when using the $S c=\infty$ technique. In some cases considerable trial and error was required to find combinations of the cycle parameters and relaxation factors which led to convergence. Most of the latest computations were undertaken on an $800 \mathrm{MHz}$ Pentium III (using just one processor) and, for reference, with that machine a typical W-cycle required about $0.112 \mathrm{~ms} /$ node. A relatively simple case $(H=10, R e=50, R i=0$, say) requiring about 20 cycles to convergence on a $674 \times 162$ grid thus took about 4 min to compute from scratch. More cycles were required for significantly higher $R e$ and/or $R i$ but few calculations required more than an overnight run.

In all cases presented here the grids were highly non-uniform because it was 


\begin{tabular}{|c|c|c|c|c|c|c|c|c|}
\hline \multicolumn{3}{|c|}{$H=10, R e=200, S c=0$} & \multicolumn{3}{|c|}{$H=10, R e=400, S c=\infty$} & \multicolumn{3}{|c|}{$H=50, R e=200, S c=1$} \\
\hline Grid & $L$ & $C_{d}$ & Grid & $L$ & $C_{d}$ & Grid & $L$ & $C_{d}$ \\
\hline $162 \times 42$ & 96.32 & 1.651 & 17 & 87.85 & 0.4 & 25 & 95.66 & 0.2607 \\
\hline $322 \times 82$ & 101.7 & 1.701 & $338 \times 82$ & 102.9 & 0.4493 & $498 \times 170$ & 103.4 & 0.2853 \\
\hline $642 \times 162$ & 102.4 & 1.713 & $674 \times 162$ & 106.9 & 0.4584 & $994 \times 338$ & 106.6 & 0.2970 \\
\hline & 102.5 & 1.717 & & 108.4 & 0.4616 & & 108.9 & 0.3073 \\
\hline
\end{tabular}

TABLE 1. Grid dependences for difficult cases. The fourth row contains values obtained by Richardson extrapolation.

particularly important to ensure adequate resolution around the plate itself. A typical smallest mesh size at the plate on the finest grids was about 0.002 . This 'nearestplate-mesh-size' was set as one of the parameters in the grid generation routine and for some cases an additional fixed mesh size was imposed at a particular downstream $x$-location, in order to minimize numerical errors in the region near the eddy closureparticularly important for the wide-wake cases (figure 2a). Mesh expansion ratios were below 1.1 everywhere, so that the additional discretization errors arising from grid non-uniformity were kept as low as possible (Castro \& Jones 1987).

In addition to speeding up the solution process, the multigrid algorithm allowed a convenient means of assessing grid dependency of solutions. This was done by interpolating the fine grid solution onto the next coarsest grid, then running the code again to convergence with that coarse grid as the finest grid. The process was repeated to obtain solutions on the successively coarser grids and Richardson extrapolation was used to estimate the errors in the finest-grid solution. It was only necessary to do this for the 'hardest' cases, i.e. flows with the largest $H$ and/or the highest $R e$ and $R i$, which typically yielded eddy lengths two orders of magnitude larger than the body scale and were thus the most taxing from a gridding point of view. We include here some examples as an indication of the general level of accuracy of our computations.

Three cases will be presented, chosen as fairly extreme cases of the three flow types illustrated in figure 2(a). Table 1 contains results for the drag coefficient and the eddy length for three successively finer grids in each of these three cases, along with the corrected values obtained by Richardson extrapolation. $C_{d}$ was computed simply by integrating the pressure difference across the plate and $L$ was deduced by appropriate interpolation of the $u$-velocity on $y=0$. For the two $H=10$ cases, the errors in $C_{d}$ and $L$ obtained on the finest grid are below $1 \%$ and $2 \%$, respectively; they are a little larger for the $H=50$ 'wide-wake' case. It should be noted that the eddy length is a particularly sensitive indicator of solution accuracy; a $2 \%$ variation can arise from a very much smaller change in the vorticity shed by the body. All the data presented in the following section are those obtained on the finest grid used for each particular case. Richardson extrapolation, whilst it could have been undertaken for all variables in all cases, would have required considerably more work and, on the basis of the results in table 1, was deemed unnecessary. In some of the more difficult cases, however, a solution on the next-coarsest grid was also obtained to check that the finest grid solution was likely to be sufficiently accurate.

In addition to grid dependency tests it was obviously important to ensure that the inlet and outlet boundaries were located sufficiently far from the body to have no effect on the solutions. This turned out to be an increasingly significant issue as $R i$ increased, as might be expected in view of the possibility of vorticity generation 
outside the eddy. For $R i=0$ it was found that even in the most extreme cases, locating the upstream boundary at $x_{u}=-60$ was quite adequate-locations further upstream resulted in changes of less that $1 \%$ in $L$ and $C_{d}$. On the other hand, for $K=0.9$ (the highest value used for most of the present computations) significantly larger upstream distances were required. Extensive tests for $H=50$, for example, demonstrated that $x_{u}=-250$ was required before further increases gave changes of less than $1 \%$. Some cases required even larger distances. The downstream boundary location, $x_{d}$, was found to be less crucial although, naturally, it needed to be significantly further downstream than the eddy closure location. Most runs had $x_{d}>2 L$. The technique employed by Ingham et al. (1990) and Ayaz \& Pedley (1999) was used to check that this was adequate. They showed that simple asymptotic solutions to equation (1.1) can be obtained for large positive (and negative) values of $x$ (for $R i=0$ ). These solutions allow the use of asymptotic boundary conditions, which were written into a separate version of the code. It was found that their use made no significant difference to the results obtained without them, provided the above condition was maintained. Since it was not straightforward to find equivalent asymptotic solutions in the $R i \neq 0$ case, we did not pursue this method for that case and relied solely on checks with larger domains for the most extreme cases.

As a final comment on solution accuracy, it is worth emphasizing the agreement between the present neutral flow results for $H=5,10$ and 20 and those obtained by Natarajan et al. (1993) using an entirely different numerical approach (they did not obtain solutions for $H>25$ ). For $50 \leqslant R e \leqslant 300$ the differences in both $L$ and $C_{d}$ were always less than $0.5 \%$. The exception was the $1.2 \%$ in $C_{d}$, for $H=5, R e=50$, but Natarajan et al. quoted their $C_{d}$ data to three significant figures only (1.46 in this case) so a significant part of the difference could well be rounding errors (the present $C_{d}$ was 1.433 for this case).

\section{Results and discussion}

\subsection{Basic data}

One of the eventual objectives is to compare results with the asymptotic behaviour outlined earlier. We start, however, with a more straightforward demonstration of the effects of $R i, R e$ and $H$ on the flow. Figure 3 shows the behaviour of $L$ and $C_{d}$ as the 'blockage ratio' $(1 / H)$ increases for a fixed value of $R e(100)$. For neutral flow $(K=0)$ the behaviour is unremarkable; as $H$ falls the eddy length falls and the drag increases (because the base pressure becomes more negative). Note again the good agreement with the result of Natarajan et al. (1993), for $H=20$. For narrow channels the qualitative behaviour when $K>0$ is similar, independent of the particular value of $K$. Results for an extreme case, $K=0.9$, are shown in figure 3. Both $L$ and $C_{d}$ are significantly smaller than for $K=0$. On the other hand, for wide channels $(1 / H<0.03)$ it is clear that the eddy length can be larger than for neutral flow, as anticipated from the asymptotic theory. Notice that, as suggested by the data of figure 3 , as $1 / H \rightarrow 0$ the results become independent of $K$, at least for the range of $K$ considered here, since a Richardson number based on body scale becomes effectively zero as $H \rightarrow \infty$. The figure includes data for $S c=1$ and $S c=\infty$ and it can be seen that the qualitative behaviour is the same and, indeed, the quantitative results are not strongly dependent on $S c$. For $S c=\infty$ the drag coefficient is noticeably higher whereas the eddy length is smaller than for $S c=0$, for all $H$. This behaviour with $S c$ was found to be quite general, so a detailed study of how the flow varies with $S c$ 

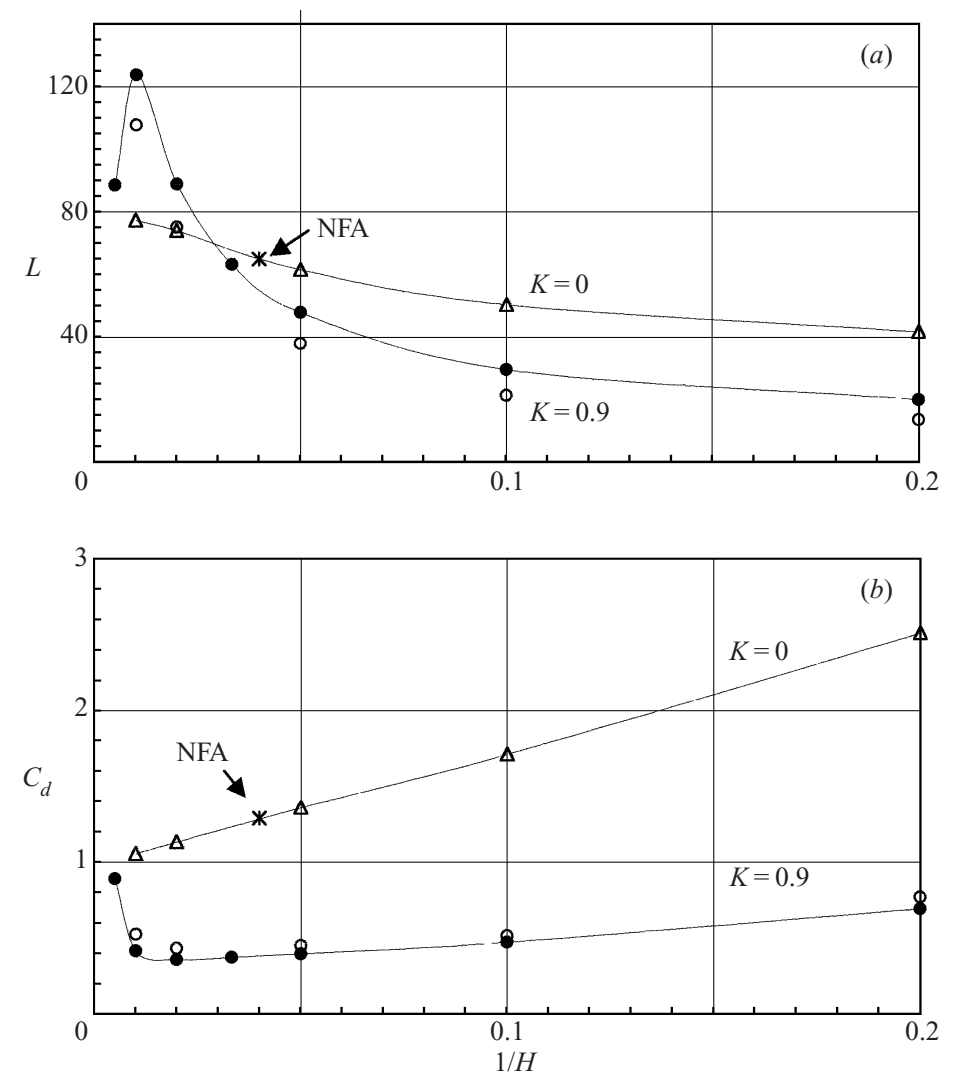

Figure 3. (a) Eddy length and ( $b$ ) drag for $R e=100 . \bullet, K=0.9, S c=\infty$; O, $K=0.9, S c=0$; $\triangle, K=0$ (i.e. neutral). $\times$, NFA, Natarajan et al. 1993.

was not performed. Although it might be of some interest to enquire what happens for $S c \ll 1$, computations were limited to the two specific cases given by $S c=1$ and $S c=\infty$, since these are representative of the most common stratifying agents that occur in practice-heat or salinity, respectively.

The flow behaviour as $K$ varies is shown for the particular case $R e=100, H=50$ in figures 4 and 5 . Recall that the theory suggests that a non-monotonic behaviour of $L$ is possible. This is demonstrated in figure $4(a) ; L$ first increases with increasing stratification before falling rapidly once $K$ exceeds about 0.8 . This fall is mirrored in the behaviour of $C_{d}$ (figure $4 b$ ). Some comments regarding the behaviour near $K=1$ are appropriate. For $K>1$, classical linear (inviscid) theory shows that obstacle drag is zero at integral multiples of $K$ but rises to a maxima in between such multiples, because of the presence of lee waves (see Baines 1995 for a helpful summary of such theories). Viscous effects and, particularly, the occurrence of separation modify this behaviour but, nonetheless, we expect a rise in $C_{d}$ as $K$ increases through unity; the data have precisely that behaviour although, because of the presence of an upstream travelling mode, converged solutions could not be obtained beyond about $K=1.04$. The existence of an essentially Kirchhoff flow on the body scale is shown by the virtually constant value of $k_{d}$, the Kirchhoff drag coefficient, which varied from 0.876 at $K=0$ to 0.906 at $K=0.98$, compared with the theoretical value of 0.88 for unbounded (neutral) flow. $k_{d}$ values were deduced from the computed solutions by 

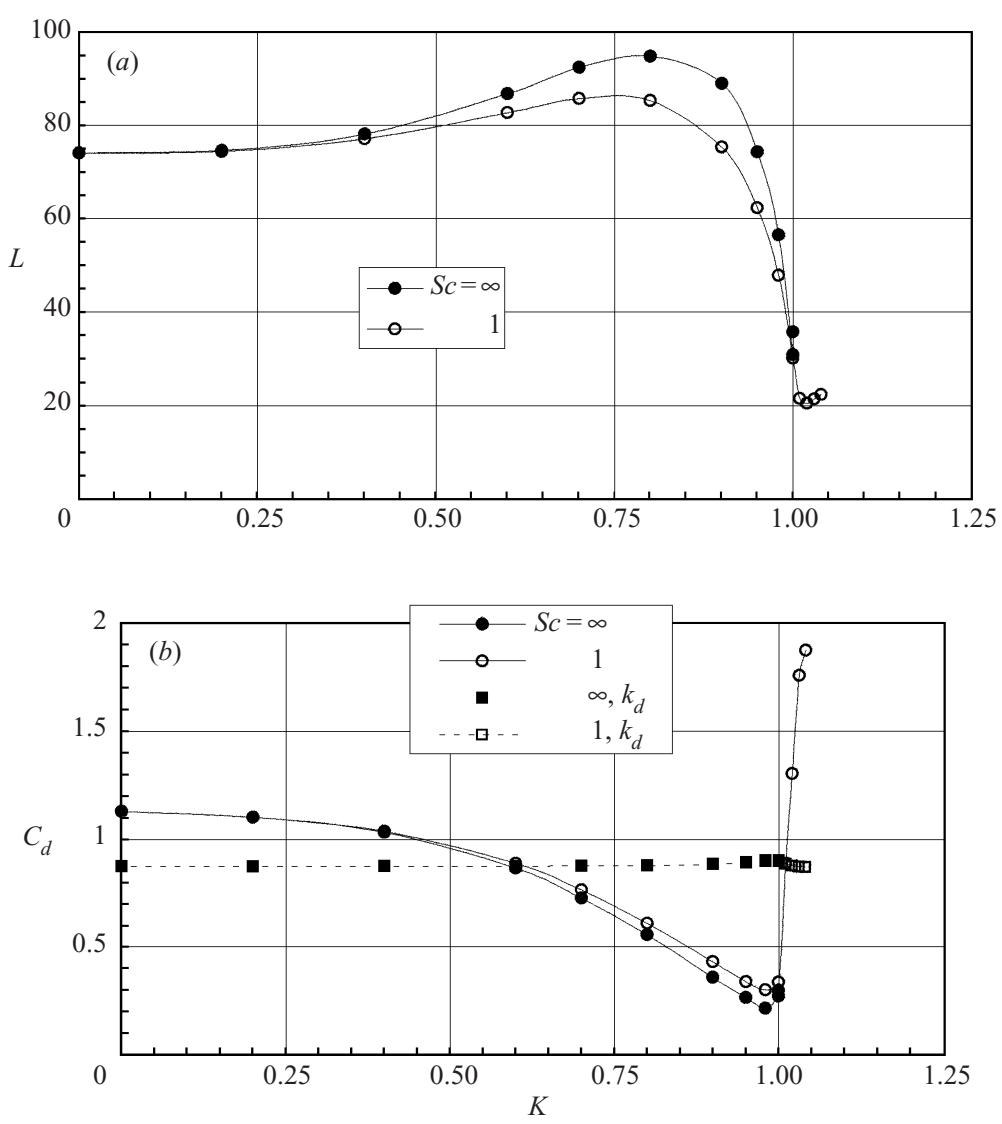

Figure 4. (a) Eddy length and $(b)$ body drag at $H=50, R e=100$. $(b)$ includes data for $k_{d}$.

using the relation $k_{d}=C_{d} /(2 \Delta P)$, where $\Delta P$ is the pressure difference between the stagnation points on either side of the plate at $y=0$. Note that for $K \geqslant 0.6, C_{d}<k_{d}$ so that the base pressure on the lee side of the plate is actually positive and the total drag is lower than the contribution from the front face, since the latter is roughly $k_{d}$.

Figure 5(a) shows the effect of stratification on the eddy width, $W$, normalized both by $L$ and $H$, and figure $5(b)$ shows the corresponding fall in eddy vorticity, $\omega_{e d}$ and rise in eddy area, $S$. For the present purposes $W$ is defined as the maximum value of $y$ along the dividing streamline and $S$ is defined as the area within this streamline (which separates from the plate at its tip and 'reattaches' at $x=L$ ). The locus of the dividing streamline was calculated as part of a second 'post-processing' version of the code, which computed values of the stream function and vorticity at each mesh point, using the previously computed velocity field and standard (first-order) methods. The eddy vorticity is taken as the average vorticity inside the dividing streamline. Increasing $K$ leads to significant increases in $W$ and hence $S$ (proportionately greater than the increase in $L$ ) and a corresponding fall in $\omega_{e d}$. Given that the vorticity generated outside the eddy diffuses into it (and is of the same sign as the eddy vorticity) it might at first sight seem surprising that stratification reduces $\omega_{e d}$. However, the velocity above the eddy near separation is reduced by the stratification (crudely, the flow finds it 'harder to go uphill') so that the shed vorticity is reduced by stratification and this effect outweighs the diffusional effect. Larger eddy areas are physically consistent with 

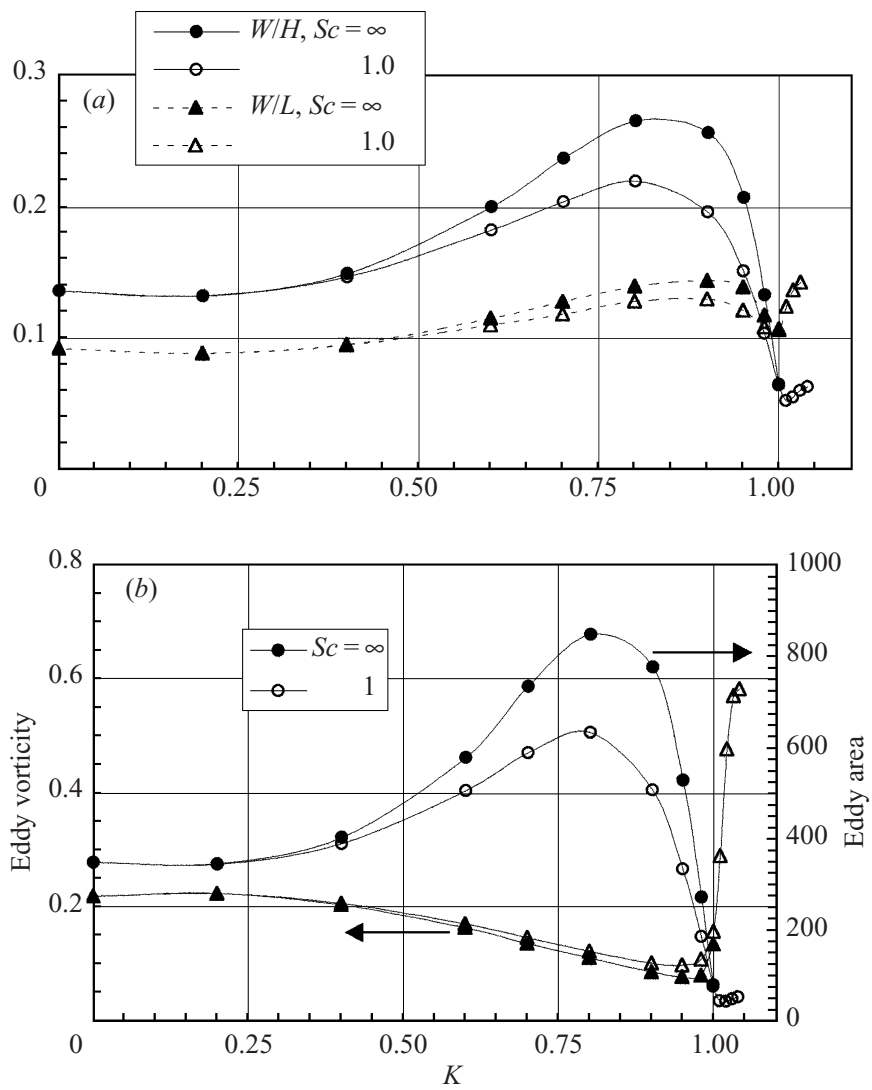

Figure 5. (a) Eddy length and $(b)$ vorticity and area.

smaller eddy vorticity since we expect, roughly, that $\omega_{e d} L=U$. Notice again that the largest effects occur for $S c=\infty$ but the behaviour is qualitatively independent of $S c$.

The influence of $R e$ at fixed $H$ and $K$ is shown in figure 6. For sufficiently small $H$ (below some critical value, $H_{c r i t}$, say) the eddy length, figure $6(a)$, simply increases linearly with $R e$ whilst the eddy width becomes roughly constant. For $K=0$ these are boundary-layer-type solutions, but it proved to be very difficult to obtain converged solutions of this type beyond a certain $R e$. (If this is symptomatic of a flow instability arising at large $R e$, it might be consistent with the finding of Milos \& Acrivos (1986) that, for a similar problem, genuine boundary-layer solutions could not be obtained if $R e$ were sufficiently large for $H$ below some critical value.) Figures $7(a)$ and $8(a)$ illustrate the wakes for such cases. Solutions with the present steady flow code could not be obtained (for $H=10$, for example) beyond about $R e=250$, although Natarajan et al. present a solution at $R e=300$ so one clearly exists. On the other hand, for sufficiently strongly stratified conditions, solutions up to $R e=500$ could be obtained (for $H=10$ ) and flows similar in appearance to the long-eddy solution of Chernyshenko (1993) appear. Figures $7(b)$ and $8(b)$ illustrate these flows, which clearly differ in character from the corresponding cases at $K=0$. In particular, the eddy closure around $x=L$ is similar in appearance to a genuine inviscid closure, i.e. it is relatively sudden, which is a specific feature of inviscid eddy closures (see Chernyshenko \& Castro 1993). However, in all cases (including the $H=5$ cases not shown here) these 'long eddies', although having an apparently inviscid closure, do not 

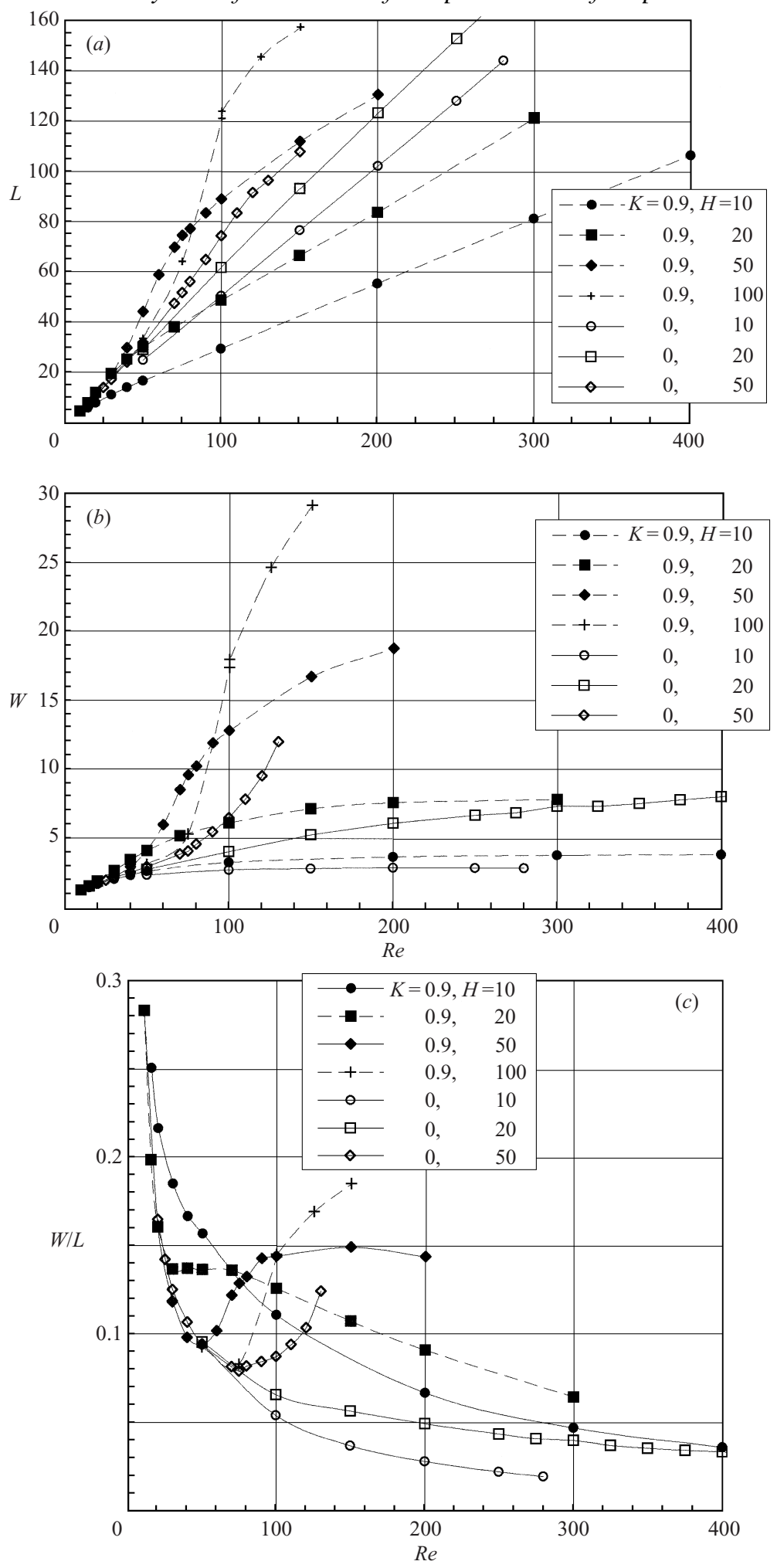

Figure 6. ( $a$ ) Eddy length and $(b)$ width for fixed $H$ and $K$.

(c) Ratio of eddy width to length for fixed $H$ and $K$. 

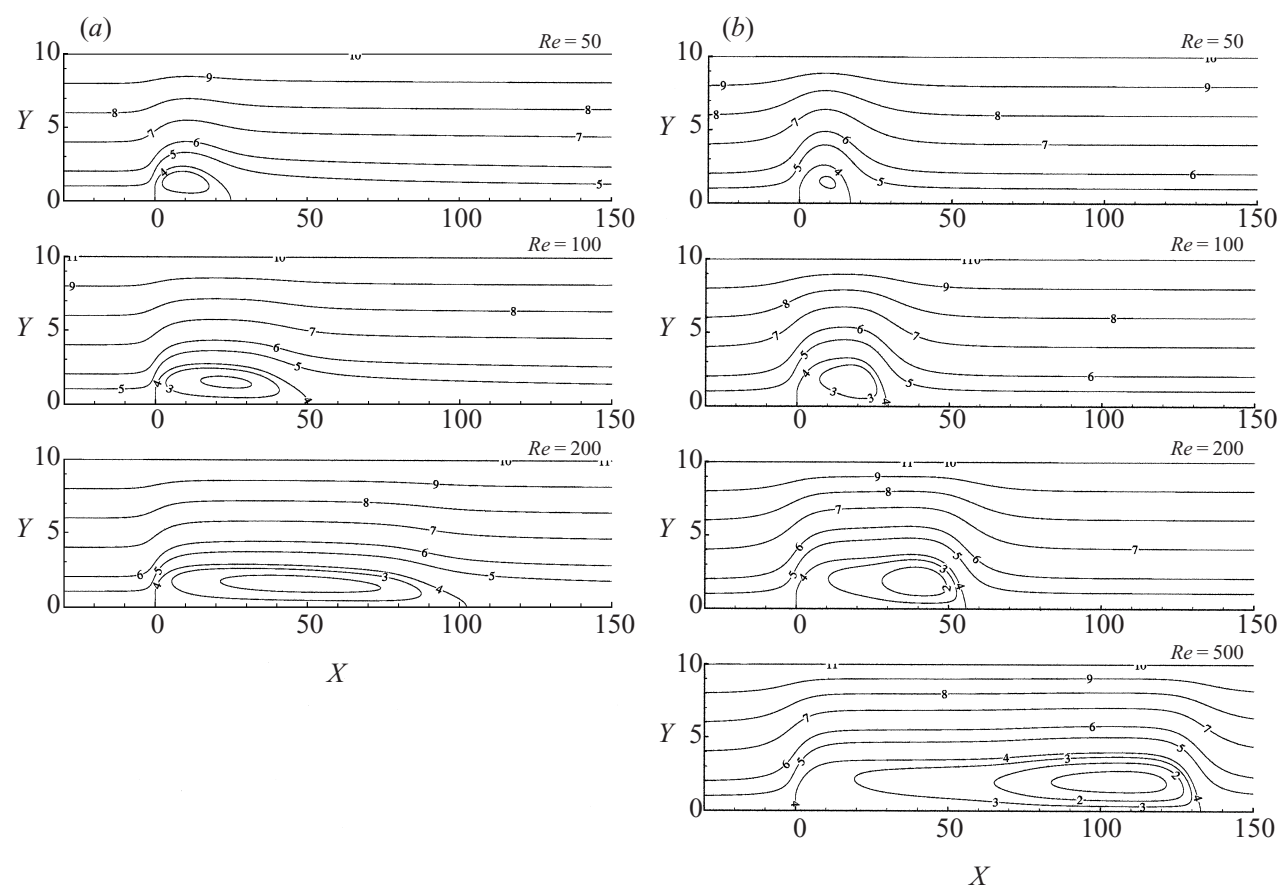

Figure 7. Streamlines for $H=10$. ( $a$ ) $K=0$ and $(b)$ 0.9. Contour values (labels): -0.6 (1), -0.4 (2), -0.2 (3), 0 (4), 1 (5), 2 (6), 4 (7), 6 (8), 8 (9), 10 (10).

have the strictly flat tops which genuine long-eddy Sadovskii eddies would exhibit; close inspection of the $R e=500$ case in figure $7(b)$, for example, shows that around $x=50$ there is a noticeable increase in eddy width. The upstream part of the eddy seems to consist of a 'development' region with the Sadovskii-type constant-vorticity, constant-width region only appearing in the latter part of the flow. This development region is more extensive than that anticipated for the 'buffer' region which $\mathrm{CC}$ pointed out must exist between the Kirchoff flow and the Sadovskii eddy. Figure 9 presents an alternative way of viewing the wakes, which highlights this feature. Vorticity surfaces are shown for a clear boundary-layer-type case $(H=10, K=0, R e=200)$, figure $9(a)$, and two flows which are more closely like the long-eddy solution, figures $9(b)$ and $9(c)$. These latter are stratified cases with $K=0.9$ and are for $R e=200$, to compare directly with figure $9(a)$, and $R e=500$. In the latter case a significant constant vorticity region is clearly developing in the rear half of the eddy, with a sudden inviscid closure, but there remains an equally long intermediate region upstream. This intermediate region does not reduce in length with increasing $R e$ so the implication is that at all $R e$ there remains an intermediate region, matching the body scale Kirchoff flow at its upstream end and the Sadovskii long-eddy flow at its downstream end. Although, as noted above, the existence of some kind of buffer region between the Kirchoff and the Sadovskii regions was suggested by $\mathrm{CC}$, its character was not delineated and the more extensive intermediate region identified here seems much more significant than was anticipated. We return to this point later.

The development of a wide wake for sufficiently large $H$ is also evident from the data in figure 6. This is most clearly seen in figure 6(c), which shows the ratio $W / L$. Once a wide wake begins to develop, the wake width starts to increase with $R e$ much more rapidly than its length so that $W / L$ is non-monotonic if $H$ is above 
(a)
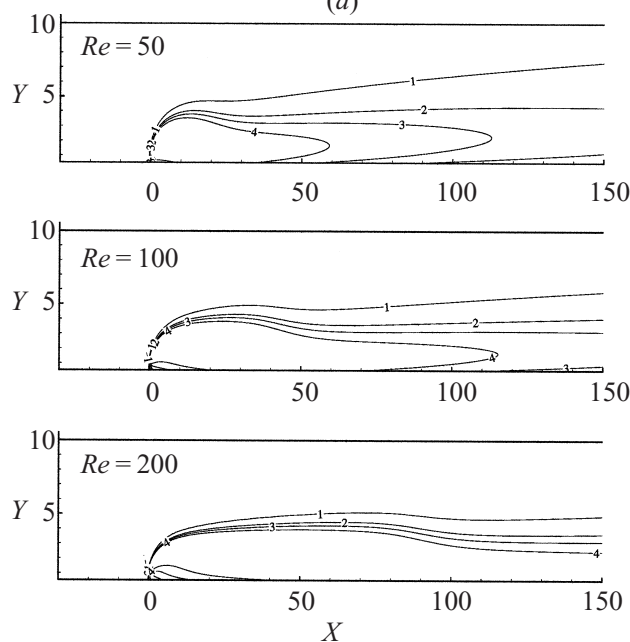

(b)
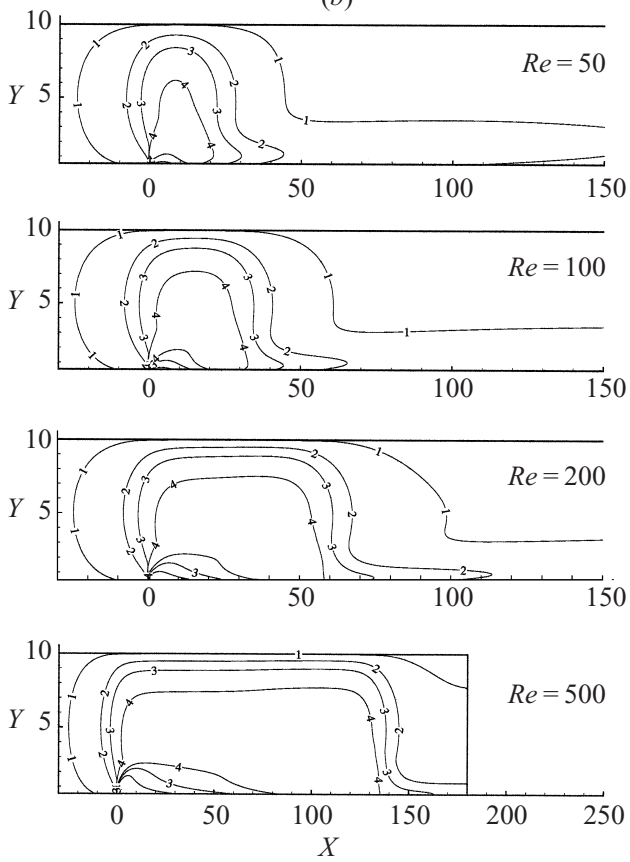

FiguRE 8. Vorticity contours for $H=10$. (a) $K=0$ and $(b) 0.9$. Levels (labels) : 0.005 (1), 0.05 (2), 0.1 (3), 0.2 (4).

$H_{\text {crit }}$. Natarajan et al. suggested that, for neutral flow, wide-wake conditions did not develop for $H<H_{\text {crit }} \approx 15$ (significantly larger than the $H_{\text {crit }} \approx 8$ found by Fornberg (1991) for circular cylinder arrays). Figure 6(c) suggests that $H_{\text {crit }}$ may be nearer 20 than 15 , since $W / L$ continues to fall monotonically for $H=20$. In fact, neutral flow computations for $H=20$ and $R e$ up to 700 were obtained and these clearly showed the development of a 'long-eddy' Sadovskii flow (rather than a wide-wake flow), separated from the body by an increasingly long intermediate region, rather like that evident in figure $9(c)$. So it could be argued that even $H=20$ is too low for genuine wide wakes, which only begin to appear incipiently before (as $R e$ increases further) being constrained by the domain to turn into long-eddy Sadovskii wakes.

For $H=50$, however, there is no doubt that wide wakes begin to appear; for neutral flow, this process seems to start around $R e=70$ whereas in stratified conditions it can appear much sooner-the $K=0.9$ data shown suggest that $R e=50$ marks the start of wide-wake development. Note that plotting the data this way (Re increasing at fixed $H$ ) implies that at sufficiently large $R e$ we expect a long-eddy solution to emerge eventually, whatever the value of $H$. So all the curves in figure $6(c)$ would be expected eventually to fall with increasing $R e$; whether or not a wide-wake flow appears first (i.e. at lower $R e$ ) depends on $H$ and $K$. It clearly does not for $H \leqslant 20$. Streamlines and vorticity contours for some typical wide wakes for the $H=50$ case are shown in figures 10 and 11, respectively. Figures 10(a) and 11(a) are for neutral flow at a Reynolds number below the point at which wide wakes start to develop, but beyond which such wakes appear for $K=0.9$, as demonstrated in figures $10(c)$ and $11(c)$. Figures $10(b)$ and $11(b)$ show a neutral flow wide wake of similar dimensions to the latter case, for $R e=120$. Figure 12 shows the corresponding vorticity surfaces 

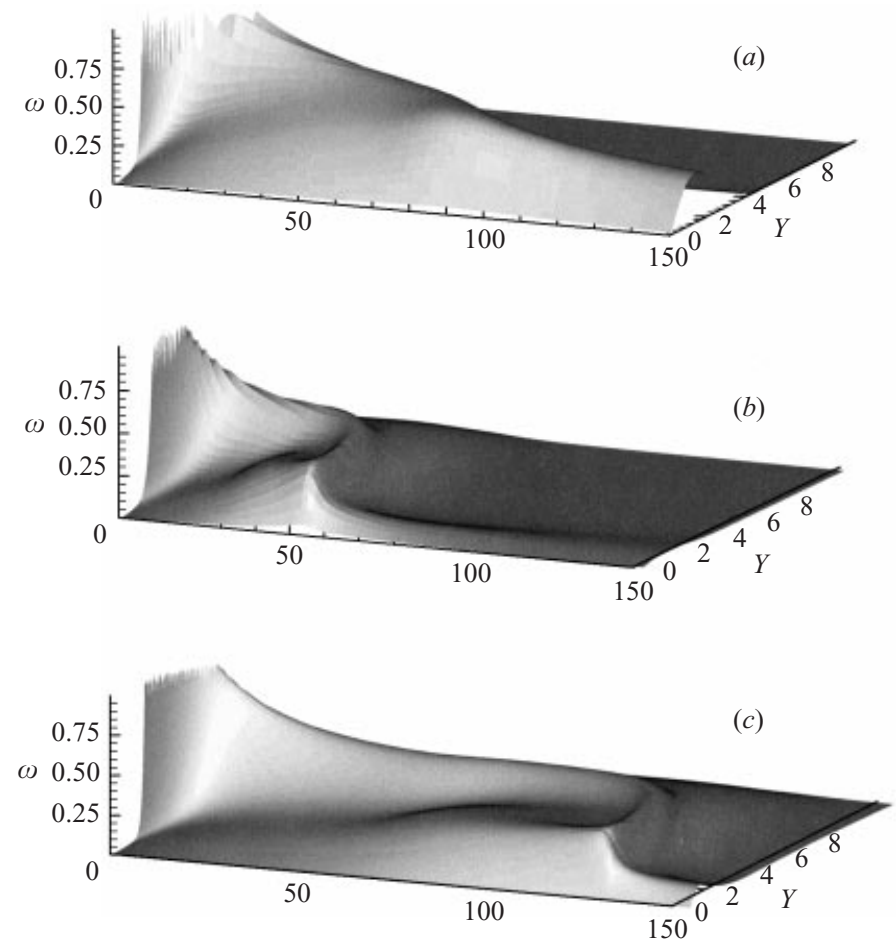

FiguRE 9. Vorticity surfaces for $H=10$. (a) $K=0$ (neutral), $R e=200$. (b) $K=0.9, R e=200$. (c) $K=0.9, R e=500$.

for these three cases; the very different character of the wide wakes (figure $12 b, c$ ) from the boundary-layer type (figure 12a) is clear. These plots also highlight the existence of the thin boundary layer (i.e. viscous) region surrounding the eddy even when the latter has a clearly wide-wake character. With increasing $R e$, this boundary layer would obviously become thinner and presumably would also reduce in (relative) amplitude until, in the limit, the vorticity plateau would simply cease in a vorticity jump (down) to zero across the dividing streamline, for cases of neutral flow.

As a further example, straightforward sequences of streamline and vorticity contour plots for increasing $R e$ at fixed $H(100)$ and $K(0.9)$ are shown in figures 13 and 14, respectively. Figure 15 shows the vorticity surfaces for the final two cases in the sequence. This sequence is a clear case of boundary-layer wakes at the lower Reynolds numbers and subsequent development of wide wakes for (in this case) $R e \approx 80$. It can be contrasted with the sequences shown in figures 7 and 8 which, because $H$ is below the critical value, do not give wide wakes at any $R e$. (It should be commented that the apparently wavy structure of the vorticity surfaces in the shear layer on the edge of the wake, visible in figure 15, is only an artefact of the plotting routine.)

\subsection{Comparisons with asymptotic theory}

Recall from $\S 2$ that the important parameters in determining the asymptotic behaviour are $H / k_{d}^{2} R e$ and $R i$, encapsulated in equations (1.8)-(1.11). In comparing the present computational data directly with the results of the asymptotic theory it is therefore appropriate to plot the data in the parametric form suggested by these equations. Before presenting such plots some comments about the expected accuracy of the theory for the parameter ranges covered here are necessary. The theory suggests 

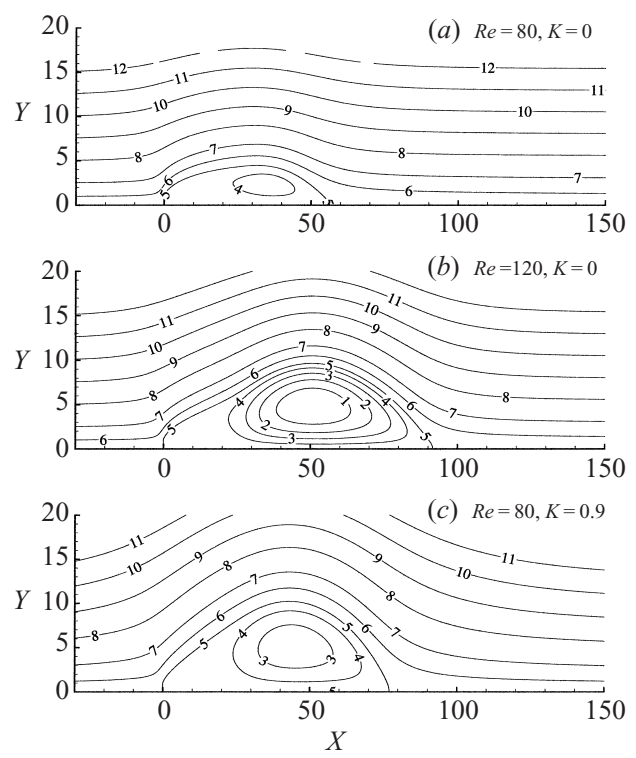

FiguRE 10. Streamlines for $H=50$. (a) $R e=80, K=0$; (b) $R e=120, K=0$; (c) $R e=80, K=0.9$; Contour values (labels): $-2(1),-1.5(2),-1$ (3), -0.5 (4), 0 (5), 1 (6), 2.5 (7), 5 (8), 7.5 (9), 10 (10), $12.5(11), 15(12)$

that the Reynolds number at which the asymptotic results may be representative of the actual flow is significantly lower in the stratified case than in the neutral case. It is shown in Chernyshenko \& Castro (1993) that the accuracy depends essentially on the value of the velocity on the free streamline on the body scale, which can be expressed by

$$
V^{2} k_{d} R e=C,
$$

where $V$ is the free streamline velocity in the Kirchoff flow (which, according to the theory, tends to zero as $R e \rightarrow \infty$ ) and $C$ is a constant. Put another way, with $V^{2}=\epsilon$, good agreement can only be expected for

$$
C /\left(k_{d} R e\right)<\epsilon
$$

with $\epsilon$ sufficiently small (lower than unity, say). In the stratified case, $C$ is a function of both $H$ and $R i$. The parametric results given by equations (1.12)-(1.15) allow curves of $\epsilon H / k_{d} v s . \epsilon k_{d} R e$ to be deduced and these are shown in figure 16, for the neutral flow case and the $K=0.9$ case. Above each curve $V^{2}<\epsilon$ and the smaller the value of $\epsilon$ the greater the accuracy of the asymptotic results. Clearly this 'acceptable' region is very much larger for $K=0.9$ than it is in neutral flow. For example, for a neutral circular cylinder flow $\left(k_{d} \approx 0.5\right)$, choosing $\epsilon=1$ suggests that, for $H=50$, an $R e$ of at least 240 is required before the flow might be expected to conform approximately to the asymptotic results. This is why only a few of Fornberg's (1991) computations for that case yield results at all comparable with the asymptotics (see Chernyshenko $\&$ Castro 1993 for detailed discussion). In contrast, for the present case of a flat plate $\left(k_{d} \approx 0.88\right)$ with $K=0.9$, the corresponding 'accuracy boundary' in figure 16 (for $H=50$ ) is crossed when $R e \approx 27$. These two cases are indicated in the figure and it is clear that the stratified cases considered here should allow better comparison with the asymptotics.

Figures 17 and 18 show the variation of $L /\left(k_{d}^{2} R e\right)$ and $C_{d} R e$, respectively, for various 

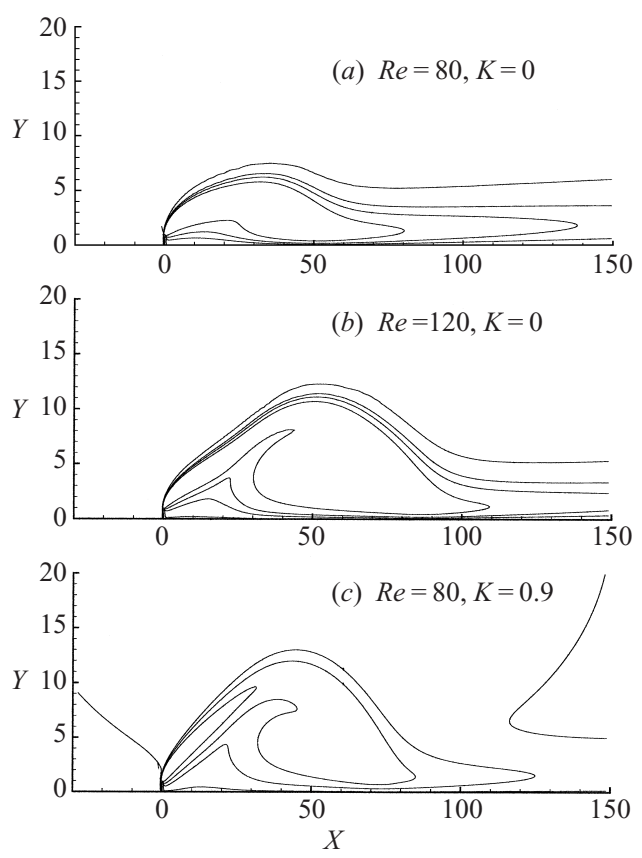

FIGURE 11. Vorticity contours for $H=50$. (a) $R e=80, K=0$; (b) $R e=120, K=0$.

(c) $R e=80, K=0.9$. Contour values (labels): 0.005 (1), 0.05 (2), 0.1 (3), 0.2 (4).

values of $H$. Since the present (and earlier) neutral flow computations arguably only just reach $H$ and $R e$ values which allow sensible comparisons with the theoretical results, discussion here is limited to the stratified cases. Only the $K=0.9$ cases are therefore included in figures 16 and 17, which also include the asymptotic results from the theory for both $K=0.9$ and for neutral conditions $(K=0)$.

Note first the behaviour indicated by the asymptotics. For $H / R e \rightarrow 0$ with $H \rightarrow \infty$ the long-eddy solution emerges, for which $C_{d}$ remains constant and $L$ increases linearly with $R e$. The theoretical curves in the figures thus have that behaviour, roughly for $H /\left(k_{d}^{2} R e\right)<0.1$. The present computational results show a very similar behaviour but, for $H=5$ and $H=10$, the numerical values of $C_{d}$ and $L / R e$ are significantly lower than those predicted. As was emphasized in $\S 1$, the asymptotic result is only valid for sufficiently large $H$ and, if $H$ is too small, the relevant 'constants' appearing in the solution $\left(\alpha_{2}\right.$ and $\gamma_{2}$ in equations (1.12) and (1.14), respectively) are likely to be functions of $H$ as well as $R i$. The present results confirm this expectation and show that increasing blockage (decreasing $H$ ) actually leads to increasing reductions in both the drag and the eddy length associated with the 'long-eddy' solution. There is as yet no theoretical solution for such cases. The apparently good agreement in eddy length (figure 17) for the $H=20$ case at the larger $R e$ is probably fortuitous; it is not mirrored in the drag data (figure 18). However, the $H=50$ data suggests that this may well be a high enough $H$ to yield close agreement with the long-eddy asymptotics, although there would have to be a two- to three-fold increase in $R e$ above what was possible with the present numerical scheme (i.e. to $\operatorname{Re}>500$ ) to be certain of this.

Secondly, the theoretical curves on these figures emphasize the point that at sufficiently large $H /\left(k_{d}^{2} R e\right)$ the effect of stratification becomes weak; unlike the $K=0$ asymptotics, there is a peak in eddy length for $K=0.9$. This occurs around 

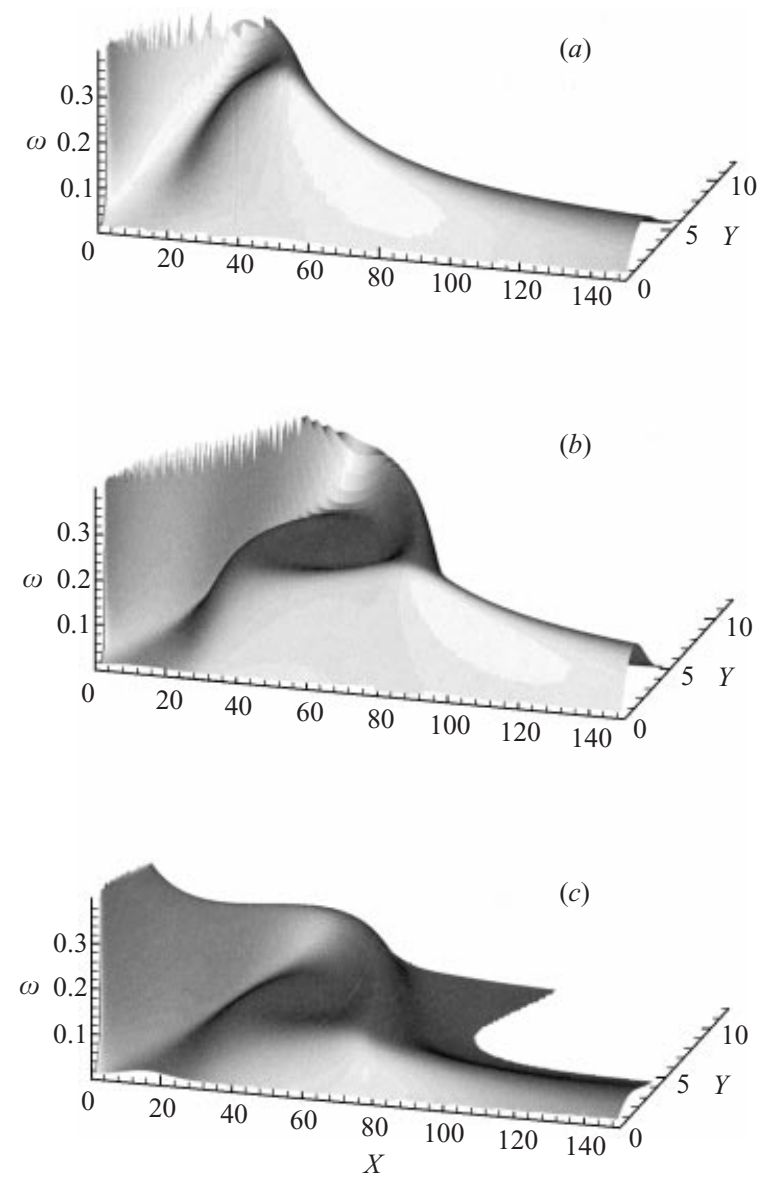

FIGURE 12. Vorticity surfaces, $H=50$. (a) $R e=80, K=0$; (b) $R e=120, K=0$;

(c) $R e=80, K=0.9$.

$H /\left(k_{d}^{2} R e\right)=4$ (figure 17) but at higher values the eddy length falls again towards the value appropriate for $K=0$. The peaks in $L$ in the numerical results in figure 17 should not be associated with this former peak. They occur simply because data at the larger $H /\left(k_{d}^{2} R e\right)$ values correspond in these computations to cases having too low an $R e$ for the flow to behave as predicted by the high $R e$ asymptotics. Similar peaks appear in the neutral flow data (not shown) and in corresponding comparisons with Fornberg's (1991) cylinder computations, as demonstrated in CC. It is interesting, however, that even at $R e$ which would seem high enough to satisfy the accuracy requirements more than adequately $(R e=100$, say) the actual flows for $H=50$ and $H=100$ have significantly higher eddy lengths and drag than the asymptotic theory suggests. Note that for $H=50$ and $H=100$ the appropriate points in the parameter space of figure 16, which are included there, are well inside the accuracy bounds, if we accept $\epsilon=1$. For $R e=100$, the boundaries are actually crossed for values of $\epsilon$ between 0.3 and 0.4 . (Alternatively, with $\epsilon=1$, the point where we expect qualitative changes in behaviour towards the asymptotic flow, the boundaries are crossed between $R e=30$ and 35.) Now if we accept the validity of the theory and thus conclude that, despite these apparently satisfied accuracy conditions, $H$ is nonetheless still not high enough to yield good agreement with it, then it is clear that 

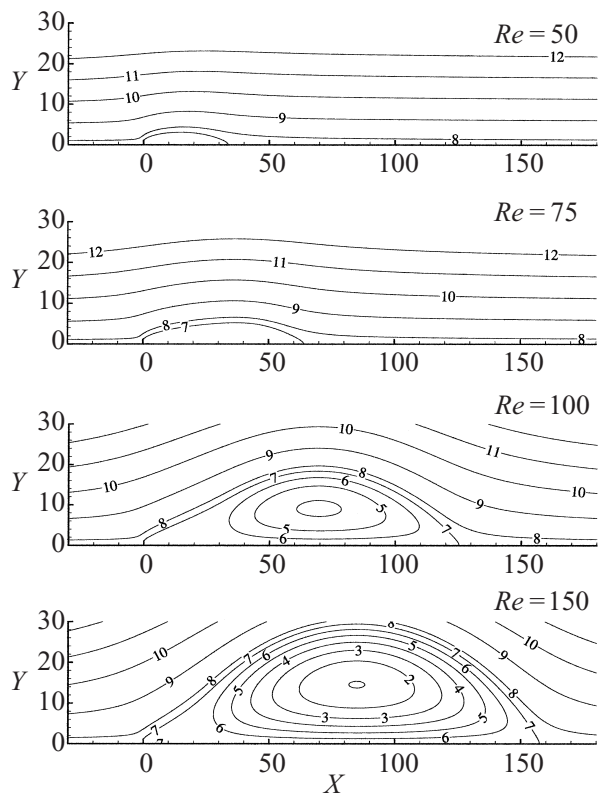

Figure 13. Streamlines for $H=100, K=0.9$. Contour values (labels): $-5.9(1),-5$ (2), -4 (3), -3 (4), -2 (5), -1 (6), 0 (7), 1 (8), 5 (9), 10 (10), 15 (11), 20 (12).
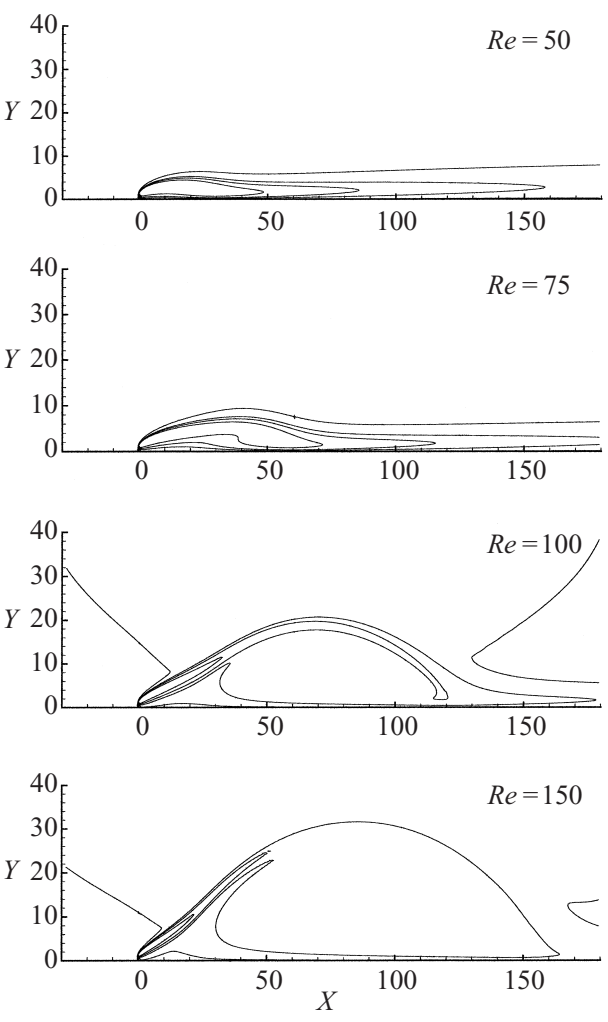

FiguRE 14 . Vorticity contours for $H=100, K=0.9$. Contour values (labels): $0.005(1), 0.05(2), 0.1(3), 0.2(4)$. 

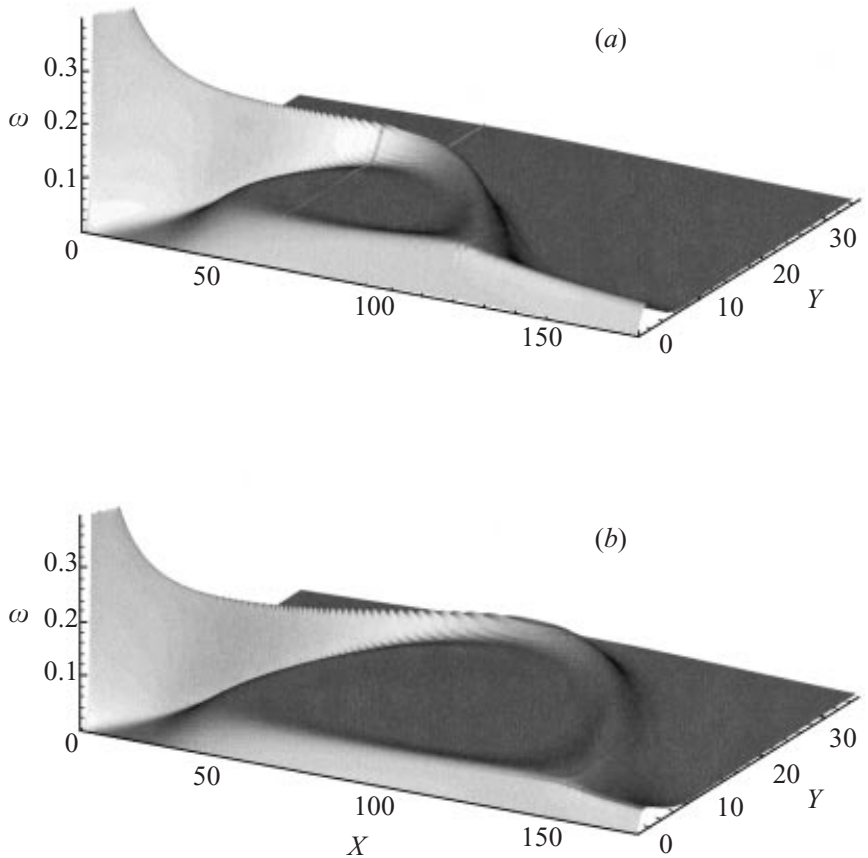

FIGURE 15 . Vorticity surfaces for $H=100, K=0.9$. (a) $R e=100$, (b) 150.

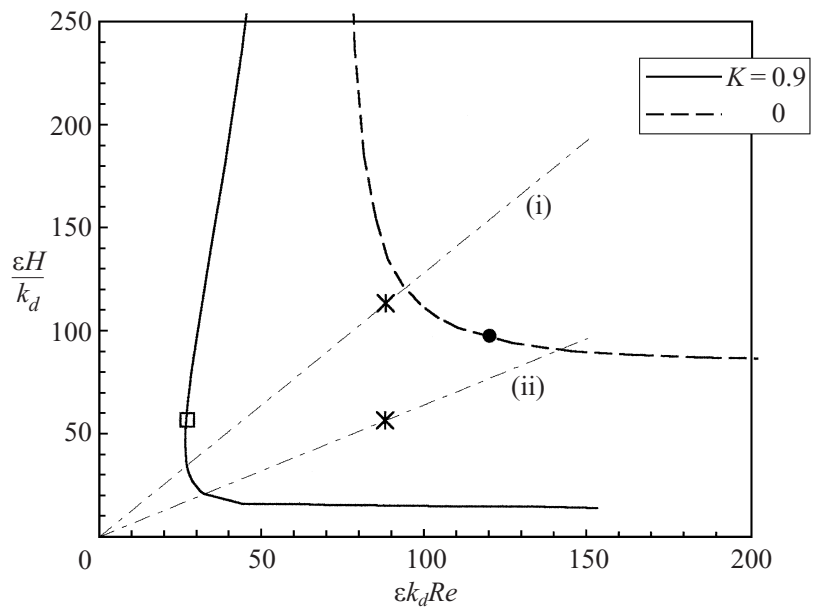

FiguRE 16. Curves of $V^{2}$ vs. $\varepsilon$ for neutral and stratified flow $(K=0.9)$. Straight lines, $H /\left(k_{d}^{2} R e\right)=$ const. for (i) $H=50, R e=100$ and (ii) $H=100, R e=100$ and include the points corresponding to $\varepsilon=1 ; \boldsymbol{\bullet}$, circular cylinder in neutral flow at $H=50, \varepsilon=1 ; \square$, flat plate at $K=0.9$.

the path towards the asymptotic result, at fixed $H /\left(k_{d}^{2} R e\right)$, cannot be monotonic with $H$. Again, there is no current theory which can account for this aspect of the flow's behaviour. Alternatively, of course, we might question the validity of the asymptotics, but there seems no good reason so to do. 


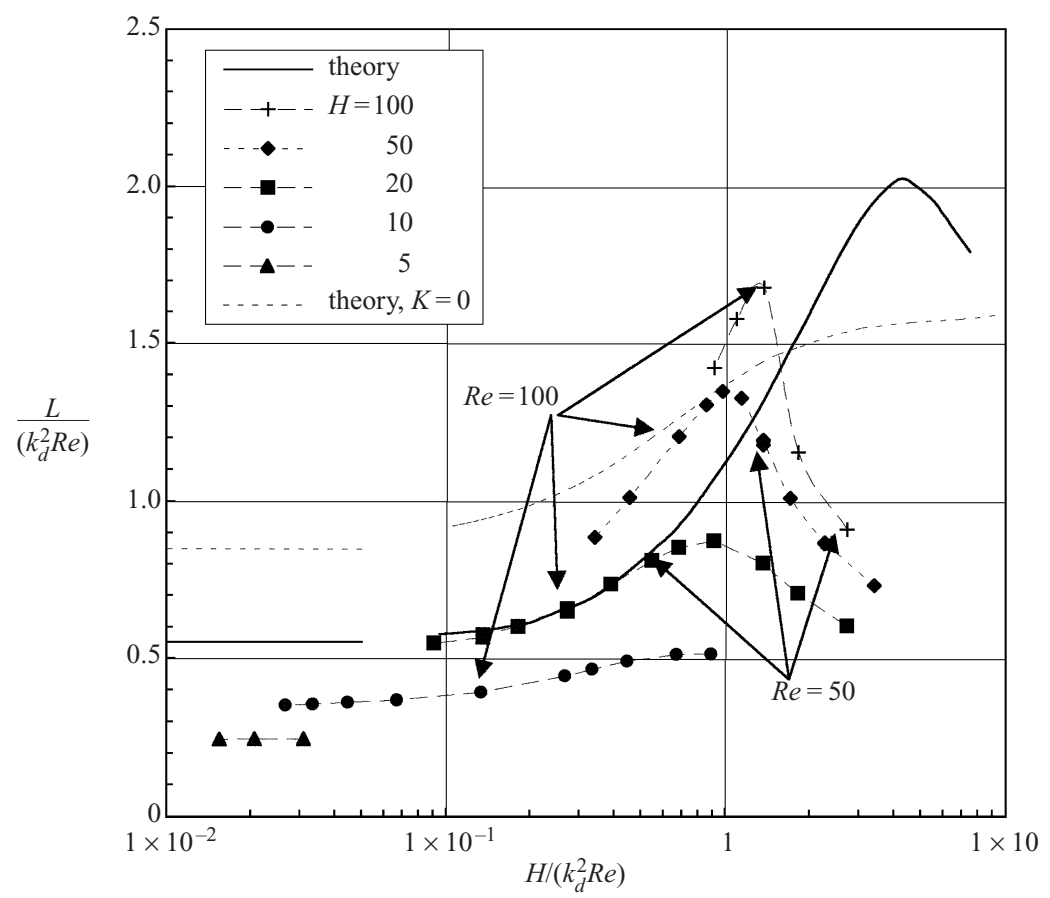

FIGURE 17. Eddy length as a function of $H /\left(k_{d}^{2} R e\right)$ for $K=0.9$.

\subsection{Non-unique solutions}

Presentation of the computational results is concluded by a brief consideration of cases in which multiple solutions appeared. Such behaviour has not been identified previously. Fornberg (1991) found that on an insufficiently fine grid, as the Reynolds number was increased the eddy length began to fall below the linear behaviour. This also occurred during some of the present computations for $K=0$. However, it also transpired that fully converged solutions could be found with a much lower $L$ than expected, in addition to the more standard solution, on precisely the same grid. Examples are shown in figure 19. Note, in particular, that use of a $340 \times 84$ grid (the second in a multi-grid sequence of three) gave two quite distinct solutions in the approximate Reynolds number range of $160<R e<190$. The initial 'lower branch' solution arose by accident, usually if too large a step in Reynolds number was attempted in computing the 'upper branch'. Once it arose, however, it could be followed both at increasing $R e$ and also at decreasing $R e$ until close to the turning point. Likewise the upper branch solution could be followed until close to its turning point. The dotted line connecting the two branches in the $L / R e$ plane (figure $19 a$ ) is a suggested (qualitative) form of the bifurcation behaviour, where the solution must presumably be unstable and therefore impossible to find with the present numerical method. (Note that the line refers to solutions on that one grid only; there is only one solution for $\operatorname{Re}>190$.)

Multiple solutions to Navier-Stokes problems written in finite-difference form have been found before in other contexts and are usually indicative of an inadequate grid and/or numerical scheme rather than genuine non-uniqueness in the NavierStokes equations. Certainly, in the present case, the point at which the bifurcation occurred depended on the grid resolution, so that the coarsest of the three grids 


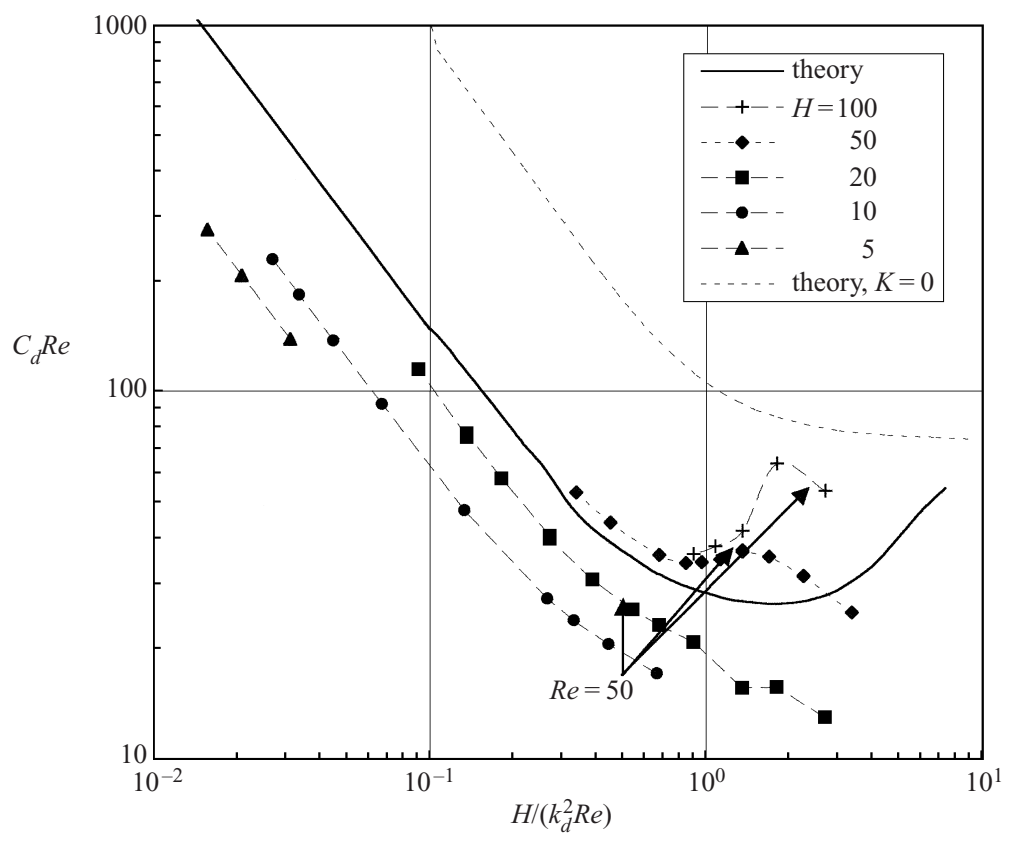

FIGURE 18. Eddy drag as a function of $H /\left(k_{d}^{2} R e\right)$ for $K=0.9$.

$(170 \times 42)$ yielded some multiple solutions at a much lower Reynolds number (and over a much smaller range), as shown in figure 19. With the finest of the three grids $(680 \times 168)$, solutions could not be obtained beyond about $R e=250$, but this was undoubtedly caused by the downstream boundary location being too near the body (at $x=180$ ). Adding additional nodes beyond this point whilst maintaining an identical grid upstream allowed converged solutions up to $R e=500$, but 'lower branch' solutions could not be found on these fine grids. This does not, of course, necessarily mean that they did not exist. It is interesting that the physical appearance of the lower branch solutions is very like the usual 'wide-wake' flow, having roughly constant eddy vorticity, as shown in figure 20, which compares vorticity surfaces for the two solutions obtained on the medium grid at $R e=175$. Furthermore, the behaviour of the lower branch solution with increasing $R e$ is what might be expected if a genuine Prandtl-Batchelor eddy structure were to be present asymptotically, i.e. eddy length tending to a constant and body drag tending to zero. The likelihood of such a flow existing at high Reynolds has been questioned previously and most recently discussed by Turfus \& Castro (2000). Their theoretical arguments suggest, however, that if such a solution were to exist asymptotically, it would only appear at Reynolds numbers significantly higher than those covered in the present work. Since, in addition, there is no doubt that the 'lower branch' solutions shown in figure 19 are grid dependent, the possible existence of a Prandtl-Batchelor solution at higher $R e$ must remain questionable. Nonetheless, these non-unique results are believed to be suggestive and a proper attack on this problem using more appropriate numerical techniques (including continuation methods, etc.) might be fruitful. At the very least, these 'lower branch' solutions serve as a salutary reminder that it is quite possible to generate converged solutions which look physically reasonable, but are nonetheless spurious. 

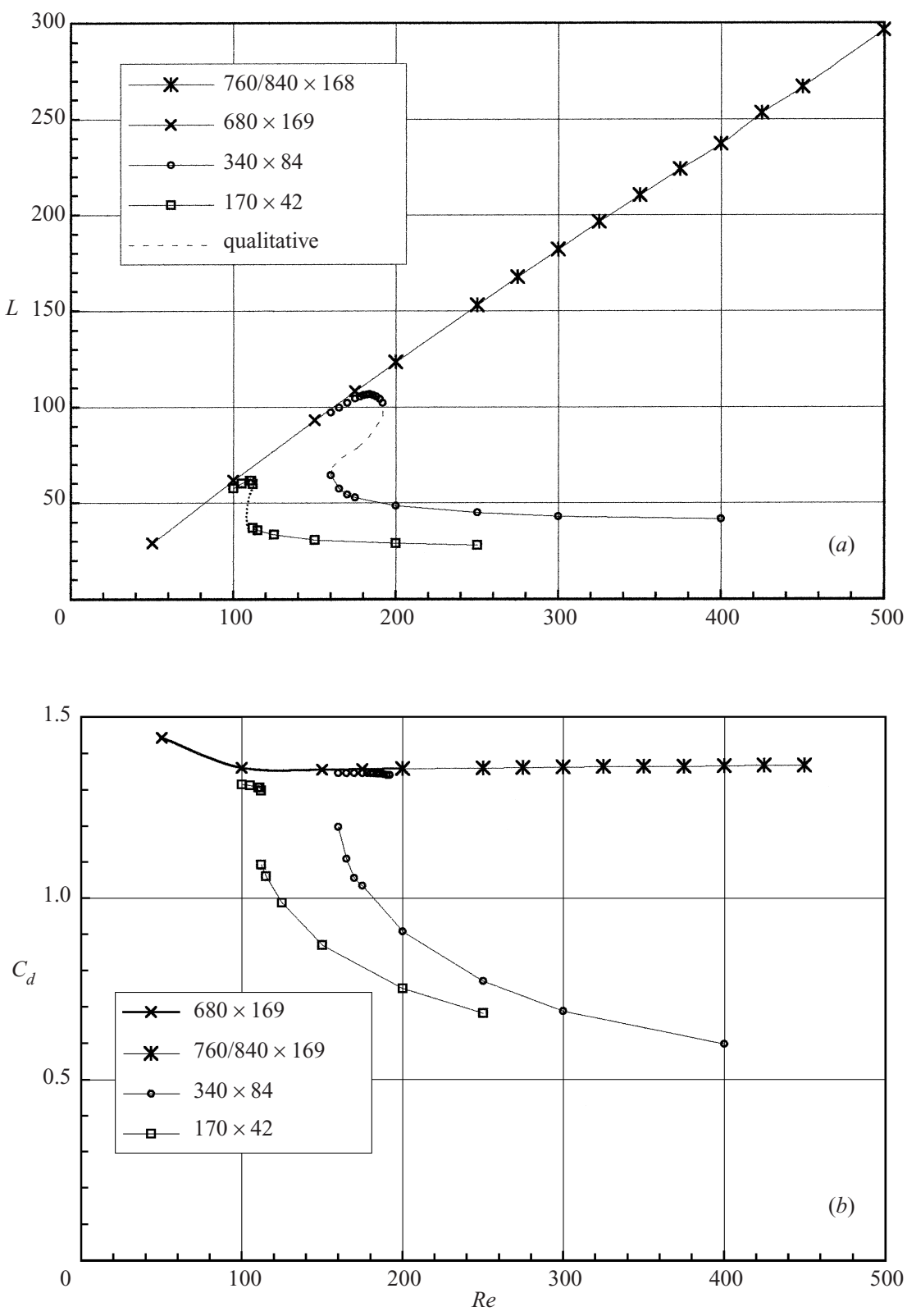

Figure 19. Examples of multiple solutions. $H=20, K=0$. (a) Eddy length and $(b)$ drag.

\section{Conclusions}

The results presented here have confirmed the qualitative behaviour suggested by the asymptotic theory of CC. For example, the theoretical result suggesting that the asymptotic structure is reached at lower $R e$ in stratified cases than in neutral cases is clearly demonstrated. However, the computed solutions have highlighted a number of distinct differences. Some of these are simply a result of the inability to reach high enough domain widths $(H)$ and/or Reynolds number $(R e)$; the asymptotics requires both to tend to infinity. For $H=50$ and $K=0.9$, for example, although the data show 

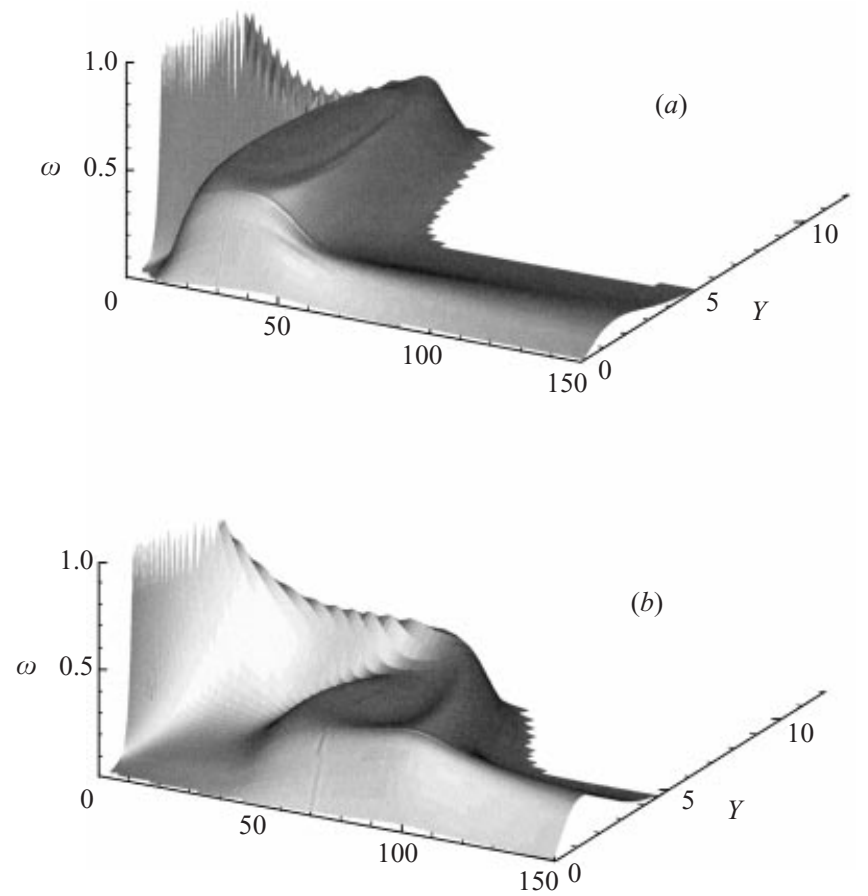

FIgURE 20. Vorticity surfaces for $H=20, K=0 . R e=175$. (a) 'Lower branch' (medium grid) solution. (b) 'Upper branch' (medium grid) solution.

the appearance of the wide-wake Sadovskii flow given by the theory, they show that quantitative agreement is likely to appear only at $R e$ considerably higher than 500 . For lower $H$ and high enough $R e$, the solutions have the appearance of the 'long-eddy' Sadovskii flow predicted by Chernyshenko (1993) (for $H / R e \rightarrow 0$ ) but, significantly, this only arises downstream of a substantial intermediate region, whose length also grows with $R e$ and appears to have a boundary-layer-like structure. There is currently no theory (asymptotic or otherwise) describing this region of the flow, which is clearly much more significant than the 'buffer' zone postulated by Chernyshenko \& Castro (1993). Furthermore, quantitative agreement with the asymptotics for this long-eddy solution does not occur, at least for $H<50$, and there is no existing theory for finite $H$ with which to compare the data.

The boundary-layer-type solutions which appeared at low $H$ (5 and 10 for neutral flow in the present set of computations) became very difficult to obtain once $R e$ exceeded about 250. This probably indicates an inherent (physical) instability in the flow, not noticed in the corresponding (neutral) computations of Natarajan et al. (1993) because they used a Newton scheme to solve the equations. In fact, subsequent time-dependent computations (to be reported separately) have indeed shown that in addition to the steady solution there exists also a periodic solution (at Reynolds numbers above about 180 for $H=10$ and neutral flow). So the steady solutions obtained for $R e>180$ are actually unstable ones. It is not yet known whether similar behaviour occurs in other cases and the whole question of stability would seem to require further investigation.

The author's colleague, Professor Sergei Chernyshenko, is gratefully acknowledged for many useful comments and discussions. Dr Martin Paisley provided helpful advice 
in regard to the most efficient use of the multi-grid aspects of the numerical code and is therefore also acknowledged with thanks.

\section{REFERENCES}

Ayaz, F. \& Pedley, T. J. 1999 Flow through and particle interception by an infinite array of closely-spaced circular cylinders. Eur. J. Mech. B/Fluids 18, 173-196.

BaInes, P. G. 1995 Topographical Effects in Stratified Flows. Cambridge University Press.

BATCHELOR, G. K. 1956 A proposal concerning laminar wakes behind bluff bodies at large Reynolds number. J. Fluid Mech. 1, 338-398.

Castro, I. P. \& Jones, J. M. 1987 Studies in numerical computations of recirculating flows. Intl J. Numer. Meth. Fluids 7, 793-823

Chernyshenko, S. 1988 The asymptotic form of the stationary separated circumference of a body at high Reynolds number. Appl. Math. Mech. 52, 746 (translated from Izv. Akad. Mekh. 52(6), 958-966.

Chernyshenko, S. 1993 Stratified Sadovskii flow in a channel. J. Fluid Mech. 250, 423-431.

Chernyshenko, S. \& CAStro, I. P. 1993 High-Reynolds-number asymptotics of the steady flow through a row of bluff bodies. J. Fluid Mech. 257, 421-449.

Chernyshenko, S. \& CASTro, I. P. 1996 High-Reynolds-number weakly stratified flow past an obstacle. J. Fluid Mech. 317, 155-178 (referred to herein as CC).

ForNBERG, B. 1985 Steady viscous flow past a circular cylinder up to Reynolds number 600. J. Comput. Phys. 61, 297-320.

Fornberg, B. 1991 Steady incompressible flow past a row of circular cylinders. J. Fluid Mech. 225, 655-671.

von Helmholtz, H. 1868 Phil. Mag. 36, 337.

Ingham, D. B., Tang, T. \& Morton, B. R. 1990 Steady two-dimensional flow through a row of normal flat plates. J. Fluid Mech. 210, 281-302.

KirChHOFF, G. 1869 J. reine angew. Math. 70, 289.

VAN LEER, B. 1974 Towards the ultimate conservative difference scheme. II Monotonicity and conservation combined in a second-order scheme. J. Comput. Phys. 14, 361-370.

LeONARD, B. P. \& MoKhtARI, S. 1990 Beyond first-order upwinding: the ultra-sharp alternative for non-oscillatory steady state simulation of convection. Intl J. Numer. Meth. Engng 30, 729-766.

Milos, F. S. \& ACRIVos, A. 1986 Steady flow past sudden expansions at large Reynolds numbers. Part I. Boundary layer solutions. Phys. Fluids 29, 1353-1359.

Milos, F. S., Acrivos, A. \& KIM, J. 1987 Steady flow past sudden expansions at large Reynolds numbers. Part II. Navier-Stokes solutions for the cascade expansion. Phys. Fluids 30, 7-18.

Natarajan, R., Fornberg, B. \& Acrivos, A. 1993 Flow past a row of flat plates at large Reynolds numbers. Proc. R. Soc. Lond. A 441, 211-235.

PAisley, M. F. 1997 Multigrid computation of stratified flow over two-dimensional obstacles. J. Comput. Phys. 136, 411-424.

Patankar, S. V. 1980 Numerical Heat Transfer and Fluid Flows. McGraw-Hill.

Peregrine, H. 1985 A note on the steady high Reynolds number flow past a circular cylinder. J. Fluid Mech. 157, 493-500.

SADOVSKII, V. S. 1970 The region of constant vorticity in plain potential flow. Uch. Zap. TsAGI 1(4), 1-9 (in Russian).

Saffman, P. G. \& TANVEeR, S. 1982 The touching pair of equal and opposite uniform vortices. Phys. Fluids A 25, 1929.

Sмiтh, F. T. 1985 A structure for laminar flow past a bluff body at high Reynolds number. J. Fluid Mech. 155, 175-191.

Taganov, G. I. 1968 Contribution to the theory of stationary separated zones. Fluid Dyn. 3, 1-11.

TAGANOv, G. I. 1970 On limiting flows of viscous fluid with stationary separation for $R e \rightarrow \infty$. Uch. Zap. Ts AGI 1, 1-14 (in Russian).

TuRfus, C. 1993 Prandtl-Batchelor flow past a flat plate at normal incidence in a channel-inviscid analysis. J. Fluid Mech. 249, 59-72.

Turfus, C. \& Castro, I. P. 2000 Proposal for a Prandtl-Batchelor solution to the problem of steady flow through a cascade of normal flat plates. Fluid Dyn. Res. 26, 181-202. 